\title{
Petrography, Geochemistry and Petrogensis of Basal Flow from Ar-Rabba Area, Central Jordan
}

\author{
Ibrahim Ahmad Ali Bany Yaseen \\ Institute of Earth and Environmental Sciences, Water, Environment and Arid Regions Research Centre, \\ Al al-Bayt University, Mafraq, Jordan \\ Email: ibanyyaseen@Yahoo.com
}

Received 17 February 2016; accepted 27 March 2016; published 30 March 2016

Copyright (C) 2016 by author and Scientific Research Publishing Inc.

This work is licensed under the Creative Commons Attribution International License (CC BY). http://creativecommons.org/licenses/by/4.0/

(c) (i) Open Access

\begin{abstract}
Thirty basaltic rock samples collected from a central Jordan at Ar-Rabba area were studied. The samples cover about $20 \mathrm{~km}^{2}$ from the Al-Rabba basalt flow. The Al-Rabba Basalt (RB) introduced Miocene to Pleistocene period. Petrography, Geochemistry and Petrogenesis investigations were carried out for the RB. The petrography analyses of the RB rocks are composed of plagioclase, pyroxene, olivine, opaque minerals (magnetite), and including secondary minerals calcite, iddingsite, and clay. The RB rocks have low range of major and trace elements concentrations, and under sodic alkaline magma series. The geochemical analysis data of RB indicated that RB was derived from a slightly fractionation magma as reflected by high $\mathrm{MgO}$ concentration range between (5.4 to $11.7 \mathrm{wt} \%$ ), and $\mathrm{Mg}$ number from 42 to 63.8, and high concentration $\mathrm{Cr}$ (18 - $385 \mathrm{ppm}$ ), Ni (160 $364 \mathrm{ppm}$ ) and low silica content ( 41.79 - $49.87 \mathrm{wt} \%)$. The chemical classification of RB is divided into basaltic, calc-alkaline to alkali basalt. The tectonic setting of RB is explained by using discrimination diagrams, Ti-Zr-Y, Ti-Zr-Sr and $\mathrm{MgO}^{-} \mathrm{FeO}_{(\text {tot) }}-\mathrm{Al}_{2} \mathrm{O}_{3}$, the $\mathrm{RB}$ plotted within the plate, calc-alkali and continental basalt respectively. The Rayleigh fractionation equation modeled for $\mathrm{Sr}$ and Ba vector diagram indicated the RB had fractionation for clinopyroxene, orthpyro-xene, olivine and trace of plagioclase.
\end{abstract}

\section{Keywords}

Calc-Alkaline Basalt, Fractionation, Tectonic Setting, Ar-Rabba, Jordan

\section{Introduction}

The regional structures of the region as a result to the tectonic evolution of the Arabian plate which includes the

How to cite this paper: Yaseen, I.A.A.B. (2016) Petrography, Geochemistry and Petrogensis of Basal Flow from Ar-Rabba Area, Central Jordan. International Journal of Geosciences, 7, 378-396. http://dx.doi.org/10.4236/ijg.2016.73030 
African and Arabian rift system, and collision of the Arabian and Eurasian plates, produce to the Arabian dome [1]-[4]. These structure evolutions led to different fracture and rifting system, i.e. East-African rift, opening of the Red Sea, and Dead Sea rift system. The tectonics evolutions were controlling to the intraplate volcanic activities in the Arabian Peninsula, which are sporadically found over an area that covers a distance of 7,000 Km from Yemen in the south through Saudi Arabia, Jordan, Syria, and up to Turkey in the north [5]-[7]. This intraplate volcanism occurred after the break of Africa and Arabia, opening the Red Sea and resulting in Oligocene flood volcanism in Yemen and Ethiopia [6] [8]. Furthermore, an extensive late Cenozoic intraplate volcanism covers the central Jordan, which may reflect the lithosphere extension [9].

This study focused on the intraplate volcanic field in central Jordan Ar-Rabba Basalt (RB). The volcanism in Jordan occurs as sporadic volcanic centers along the eastern side of the Jordan Rift (Figure 1). These volcanoes are clearly associated with continental rifting and inception of the Dead Sea plate boundary. The relationship between magmatism and tectonic of the intraplate volcanism has been reported by [10], indicating that alkaline volcanism in Jordan is similar to the Arabian intraplate volcanic fields, which erupt through two main fissure systems along the eastern margin of the Dead Sea rift in the east-west direction [11] [12]. The volcanism might have probably commenced during the Miocene period and continued to Pleistocene [13]. The K-Ar age of Jordan basaltic activity is divided into three episodes: Oligocene to early Miocene (26.23 - 22.17 Ma), Middle to late Miocene (13.97 - 8.94 Ma), and late Miocene to Pleistocene (6.95 - $0.15 \mathrm{Ma}$ ) [14]-[16]. The Jordan basalt is distributed in three main regions based on their mode of occurrences, within Jordan rift (e.g. Zara basalt), central Jordan (e.g. El-Lajjun, Ar-Rabba (Shihan) basalt and NE-Jordanian Harrat (with an area 11,400 $\mathrm{km}^{2}$ ) which is a part of the largest Harrat Al-Shaam and NW Jordan Ash-Shuna Ash-Shamaliyya Area [17]-[19].

The basalts in central Jordan have been found to occur in seven places, namely, Tafila, Wadi Dana, Jabel Shihan (RB), El-Lajjoun, Jurf Al-Darawish, Ghor Al-Katar, and Wadi Zarqa-Ma'in, in the form of plateau basalts, local flows (wadi fills), or individual volcanic bodies (cones, plugs, and dikes) [19]. Some doloritic dykes observed to occur along the major faults, such as Karak-Al-Fayha fault zone, Wadi Dana, Tafila, and Ar-Rabba plateau [13]. The objective of this study is to investigate the petrography, geochemistry, and petrogenesis evolution of the intercontinental basaltic flow at Ar-Rabba area, which is in the east of Dead Sea in central Jordan; to determine the origin and type of the parental magma; and to investigate the tectonic setting evolution of the RB region.

\section{Geology Setting}

The RB is located about $13 \mathrm{~km}$ Northeast of Al-Karak city at $31^{\circ} 15^{\prime} 3468$ to $31^{\circ} 15^{\prime} 3488 \mathrm{~N}$ and $35^{\circ} 30^{\prime} 752$ to $35^{\circ} 30^{\prime} 760 \mathrm{E}$ (Figure 1). The RB flow covers an area of about $20 \mathrm{~km}^{2}$ with arc like strip width about 0.2 to 0.5 $\mathrm{km}$ with an average thickness $1-5 \mathrm{~m}$ [13]. The exposure strata along the sides of Wadi Abu-Jubayba, Wadi ad Dahalya, Wadi imra, Wadi ad Dafall, Wadi al Hidan and Wadi adh Dhira area. This basalt belongs to the middle Pleistocene and consistence with the second stage of the opening of the Red Sea over the last 5 Ma [17] [20]. The RB seen in the field cover by Al-Hisa Phosphorite unite (Belqa Group) of Campanian age [21] [22], and coverd by fluviatile, lacustrine gravels and soil.

The study area is affected by different structures, such as Siwaqa fault with east-west direction and extends from the Dead Sea to Wadi Sirhan, and Ed dhira fault are two major trending NE-SW. The N-S trending fractures are parallel to the Dead Sea transform fault, where as the E-W fractures lie parallel to Siwaqa fault Hasa fault and Zarqa-Ma'in fault [23].

\section{Sampling and Analytical Techniques}

A total of 30 representative rock chip samples were collected from the outcropping AR-Rabba Basalt (RB). The samples crushed and powdered using a stainless steel Jaw Crusher and an Agate Ball Mill machine, to obtain grain size less than $-80 \mu$. The samples were quartered in order to get a statistically representative (splitter) fraction and powdered using two geochemical techniques at the Natural Resources Authority (NRA) labs.

The major elements were analyzed on fused glass discs-like pellet (bead) by using a Phillips X-Ray Florescence Spectrometry (XRF) Majex PW-2424 Model at the Al al-Bayt University. A total of 2 gram of the powder samples were mixed with 8 gram of lithium tetra borate and fused in platinum crucibles over gas burners $\left(1000^{\circ} \mathrm{C}\right)$ for $1 \mathrm{~h}$. The melts were poured into a mold to create glass disks. The Loss on Ignition (LOI) was determined by the weight lost after melting at $1000^{\circ} \mathrm{C}$. 


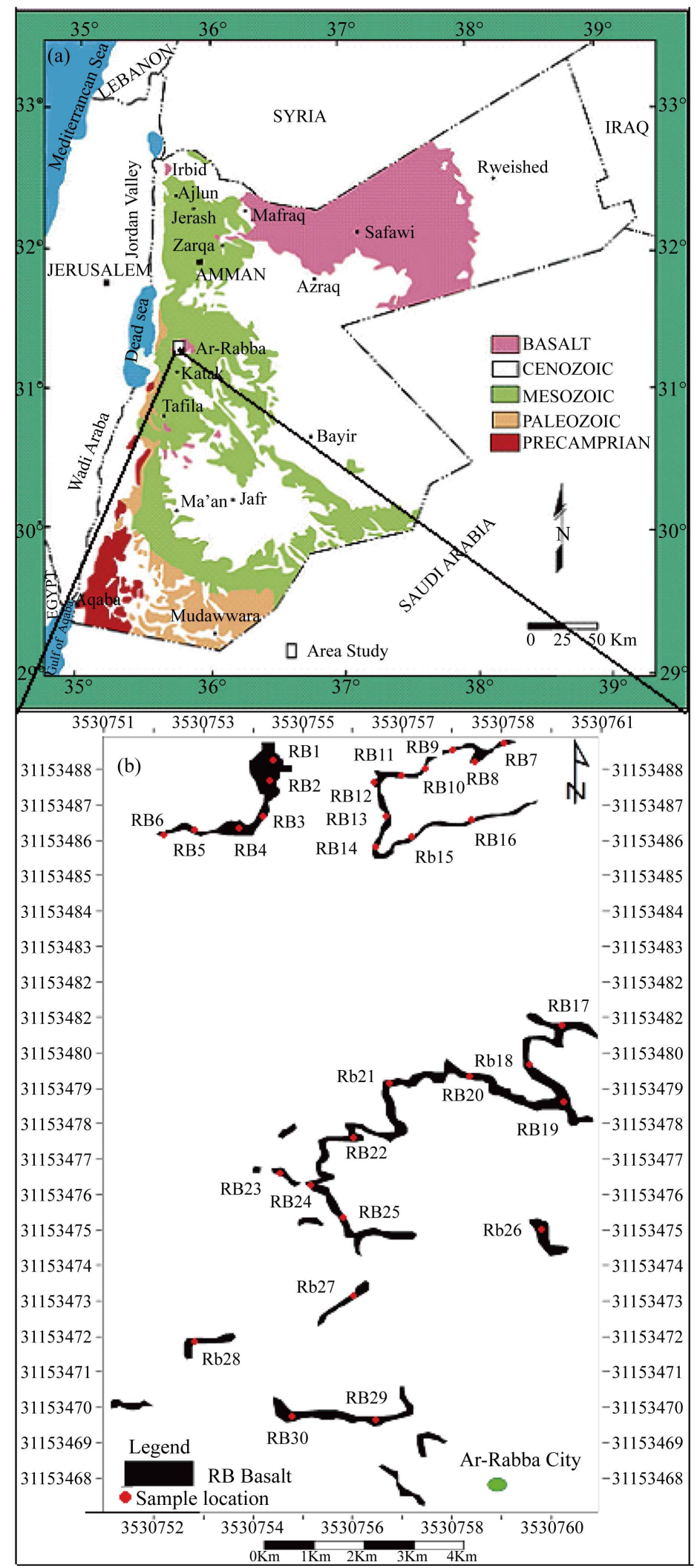

Figure 1. (a) Simplified Geological map of Jordan show the area study; (b) Geological and sample location map of the area under study after [25]. 
The trace elements were analyzed by decomposition using Ione Inductively Conductive Coupled Plasma Emission Spectroscopy (ICP-AES) at Natural Resources Authority Labs. A total of 1 gram of the powdered $<80$ $\mu$ sample mixed with 3 gram of sodium peroxide $\left(\mathrm{Na}_{2} \mathrm{O}_{2}\right)$ were placed in a zirconium crucible, and fused by heating it to $450^{\circ} \mathrm{C}$ for 45 mints, to obtain a sinter. Subsequently, $72 \mathrm{ml}$ of deionized water was added to it, and stirred for a few mints; then $28 \mathrm{ml}$ of diluted $\mathrm{HCl}$ in a ratio 1:1 was added to obtain clear solutions that were used to determine the trace element concentrations. Thin sections were prepared at the University of $\mathrm{Al}$ al-Bayt and petrographically investigated via polarizing microscope type Lico proccer with different magnifications.

The geochemical data were processed and pictorially represented by using the computer program Igpet 32 . CIPW-Norm calculations were carried out by using the Excel sheet [24].

\section{Results}

\subsection{Petrography and Mineralogy}

The general description of the 30 rock samples for RB, is melanocritic, holocrystalline, hypidiomorphic, intergranular fine to medium grained, exhibited aphentic to porphyritic, glomeroporphyritic, vesicular or amgdaloidal texture. The mineral constituents were plagioclase, olivine, pyroxene and opaque minerals (magnetite). The secondary minerals included iddingsite, calcite, serecite and clay. The common texture of the RB was trachyt, glomeroporphyritic, vesicular, amygdaloidal, seriate, and ophtic to subophtic texture (Figure 2).

\subsubsection{Plagioclase}

Plagioclase crystals are forming more than 40 vol\% for modal value of the basaltic phenocrysts and miro-phenocrysts in the ground mass, and average 49.08 vol\% for norm analysis (Table 1). They occur in variety of forms and size, subhedral to anhedral, and range in size from medium to fine phenocrysts to very fine acicular to needle microlites in the groundmass. The crystals are showing simple twining and normal zoning (Figure 2(a)). The extinction angles on plagioclase phenocrysts ranged between $15^{\circ}$ to $22^{\circ}$, indicating to oligoclase to andesine composition (An30 - An40), determined by using [26] method. Plagioclase crystals are clear in plane polarized light (PPL) and gray interference color in XPL and slightly withered to calcite. The elongated slight alignment plagioclase crystals exhibited orientation to olivine and pyroxene crystals, presenting a trachytic texture (Figure 2(b)). Some of the plagioclase crystals enclosed in pyroxene occurs ophitic to supophitic texture (Figure 2(c)). The glomerphyrritic texture occurs with associated plagioclase, pyroxne and olivine are enclosed in fin-grained ground mass in clusters of four crystals (Figure 2(d)).

\subsubsection{Pyroxene}

The clinopyroxene (cpx) is the second dominant mineral in RB, form more than $30 \mathrm{vol} \%$. It is occurred as colorless at (PPL), and second to their order interference color (XPL) with subhedral to anhedral. The crystal had size between ( 2 to $6 \mathrm{~mm}$ ), with perfect two set cleavage intersect at $\sim 90^{\circ}$ in the cross-section. The clinopyroxene had an inclined extinction, indicating the presence of clinopyroxene of diopside. The normative RB indicating to the diopside content was average 22.5 vol.\% (Table 1). The clinopyroxene intersected with plagioclase crystals to form ophitic to subophtic texture (Figure 2(c)), and the crystal zoning was present in clinopyroxene (Figure $2(\mathrm{e}))$.

\subsubsection{Olivine}

The olivine phenocrsts are occurred subhedral to anhedral crystas, and up to $3 \mathrm{~mm}$ in diameter in the groundmass and forming morthan $20 \mathrm{vol} \%$ for modal and average 6.48 for norm (Table 1). The olivine crystals were colorless and displaying seriate texture with high degree of alteration to iddingsite (Figure 2(f)). The aggregate crystals exhibit glomeroporphyritic texture. Iddingtization was common particularly the edge (rim) and fracture of the crystals, and some crystals were partially to completely pseudomorphosed to brown iddingsite (Figure $2(\mathrm{~g})$ ). The alteration produced a result from relatively low temperature deuteric alteration process [19], and the embayment of olivine crystals as a result of interaction between melt and olivine crystals during the crystallization process (Figure 2(g)) [27].

\subsubsection{Opaque and Secondary Minerals}

Opaque minerals were commonly found in RB, forming about $10 \mathrm{vol} \%$ of the rocks and ranging from 0.5 to 4 

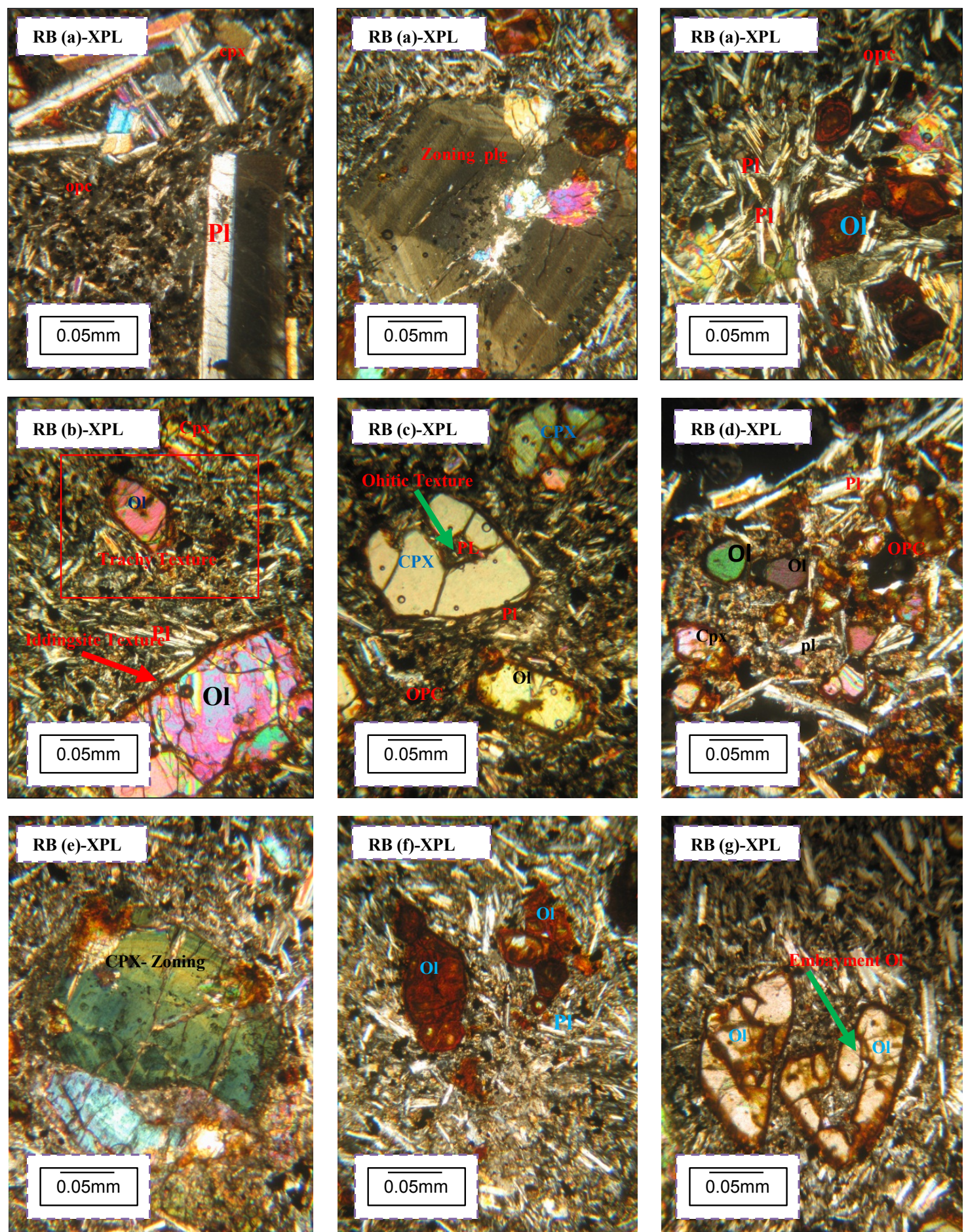

Figure 2. Photomicrographs of the RB studied. (a) Simple twining and zoning of plagloclase, with intergranular texture and opaque minerals (XPL, magnification $4 \times$ ); (b) Tarchy texture with subhedral olivine crystal with high iddingsite altration and showing opaqu minerals (XPL, magnification 4×); (c) Subhedral clinopyroxene crystals intersected with plagioclase crystals to form ophitic to subophtic texture (XPL, magnification 4×); (d) Glomerphyrritic texture occurs with associated plagioclase; pyroxene and olivine enclosed in fin-grained ground mass and shows opaque minerals (XPL, magnification $4 \times$ ); (e) Zoning of clinoyroxene crystal (XPL, magnification $4 \times$ ); (f) Seriate texture with high degree of alteration olivine to iddingsite (XPL, magnification $4 \times$ ); $(\mathrm{g})$ Embayment of olivine crystals with highly alteration to iddingsite. Ol: olivine; cpx: clinopyroxene; Pl: plagioclase; Opc: opaque minerals; magnification $4 \times=0.05 \mathrm{~mm}$ ). 
Table 1. Chemical composition of the samples from RB. The major oxides are given in wt $\%$, trace elements in ppm and CIPW-wt \% norm.

\begin{tabular}{|c|c|c|c|c|c|c|c|c|c|c|}
\hline S. No. & $\mathrm{Rb} 1$ & $\mathrm{Rb} 2$ & $\mathrm{Rb} 3$ & $\mathrm{Rb} 4$ & $\mathrm{Rb} 5$ & $\mathrm{Rb} 6$ & $\mathrm{Rb} 7$ & $\mathrm{Rb} 8$ & Rb9 & $\mathrm{Rb} 10$ \\
\hline $\mathrm{SiO}_{2} \mathrm{wt} \%$ & 44.9 & 47.6 & 45.98 & 45.97 & 45.41 & 46.68 & 47.46 & 46.57 & 47.3 & 46.23 \\
\hline $\mathrm{TiO}_{2}$ & 2.8 & 2.5 & 2.1 & 2.76 & 2.44 & 2.6 & 1.3 & 1.6 & 1.63 & 1.44 \\
\hline $\mathrm{Al}_{2} \mathrm{O}_{3}$ & 12.3 & 12.0 & 9.6 & 11.9 & 11.97 & 12.5 & 12.8 & 13.6 & 13.33 & 13.24 \\
\hline $\mathrm{Fe}_{2} \mathrm{O}_{3}$ & 13.1 & 12.1 & 10.4 & 12.7 & 11.8 & 11.8 & 10.8 & 12.9 & 13 & 11.8 \\
\hline $\mathrm{MnO}$ & 0.22 & 0.23 & 0.18 & 0.21 & 0.26 & 0.21 & 0.17 & 0.19 & 0.19 & 0.17 \\
\hline $\mathrm{MgO}$ & 10 & 10.6 & 8.7 & 10.3 & 11.1 & 9.77 & 9.6 & 8.9 & 9.98 & 8.3 \\
\hline $\mathrm{CaO}$ & 9.7 & 10.1 & 17.8 & 10.3 & 10.2 & 9.98 & 10.9 & 9.4 & 9.3 & 12.85 \\
\hline $\mathrm{Na}_{2} \mathrm{O}$ & 3.43 & 2.76 & 1.97 & 2.86 & 3.12 & 3.22 & 3.54 & 2.85 & 2.47 & 3.34 \\
\hline $\mathrm{K}_{2} \mathrm{O}$ & 0.97 & 1.2 & 0.94 & 0.81 & 1.07 & 0.9 & 0.64 & 1.62 & 0.63 & 0.87 \\
\hline $\mathrm{P}_{2} \mathrm{O}_{5}$ & 0.24 & 0.28 & 0.32 & 0.41 & 0.52 & 0.47 & 0.39 & 0.53 & 0.73 & 0.32 \\
\hline LOI & 1.36 & 1.17 & 1.64 & 1.84 & 2.21 & 2.24 & 2.32 & 1.95 & 2.12 & 1.77 \\
\hline Sum & 99.02 & 100.54 & 99.63 & 100.06 & 100.10 & 100.37 & 99.92 & 100.11 & 100.7 & 100.33 \\
\hline $\mathrm{Mg} \#$ & 60.2 & 63.4 & 62.9 & 61.6 & 63.5 & 62.1 & 63.8 & 57.8 & 60.3 & 58.2 \\
\hline $\mathrm{Na}_{2} \mathrm{O} / \mathrm{K}_{2} \mathrm{O}$ & 3.4 & 2.16 & 2.1 & 3.21 & 2.92 & 3.58 & 5.53 & 1.76 & 3.92 & 3.84 \\
\hline $\mathrm{Al}_{2} \mathrm{O}_{3} / \mathrm{TiO}_{2}$ & 4.39 & 4.8 & 4.57 & 4.31 & 4.6 & 5 & 9.84 & 8.5 & 8.18 & 9.19 \\
\hline Cr ppm & 321 & 384 & 293 & 360 & 385 & 292 & 225 & 286 & 296 & 270 \\
\hline $\mathrm{Co}$ & 56 & 53 & 43 & 50 & 48 & 38 & 21 & 40 & 40 & 46 \\
\hline $\mathrm{Ni}$ & 248 & 286 & 228 & 273 & 295 & 210 & 214 & 275 & 280 & 273 \\
\hline $\mathrm{Cu}$ & 63 & 65 & 61 & 58 & 49 & 43 & 63 & 69 & 69 & 64 \\
\hline $\mathrm{Zn}$ & 126 & 118 & 100 & 124 & 117 & 118 & 84 & 102 & 104 & 96 \\
\hline $\mathrm{Sr}$ & 796 & 812 & 683 & 1019 & 1093 & 700 & 534 & 631 & 626 & 561 \\
\hline $\mathrm{Y}$ & 19 & 19 & 15 & 19 & 18 & 18 & 13 & 16 & 16 & 15 \\
\hline $\mathrm{Nb}$ & 63 & 58 & 48 & 63 & 55 & 55 & 12 & 18 & 18 & 19 \\
\hline $\mathrm{La}$ & 36 & 35 & 25 & 33 & 34 & 32 & 13 & 21 & 21 & 19 \\
\hline $\mathrm{Ce}$ & 57 & 59 & 47 & 56 & 52 & 41 & 32 & 12 & 14 & 14 \\
\hline $\mathrm{Pb}$ & 3 & 2 & 5 & 4 & 5 & 3 & 6 & 14 & 9 & 6 \\
\hline $\mathrm{Ba}$ & 439 & 385 & 263 & 472 & 364 & 471 & 251 & 196 & 198 & 412 \\
\hline $\mathrm{Zr}$ & 298 & 294 & 311 & 298 & 250 & 210 & 117 & 175 & 159 & 183 \\
\hline $\mathrm{Cr} / \mathrm{Ni}$ & 1.29 & 1.34 & 1.28 & 1.31 & 1.3 & 1.39 & 1.05 & 1.04 & 1.05 & 0.98 \\
\hline $\mathrm{Zr} / \mathrm{Nb}$ & 4.73 & 5.06 & 6.48 & 4.73 & 4.54 & 3.81 & 9.75 & 9.72 & 8.83 & 9.63 \\
\hline $\mathrm{Zr} / \mathrm{Y}$ & 16.55 & 15.47 & 20.73 & 15.68 & 13.88 & 11.66 & 9 & 10.93 & 9.93 & 12.2 \\
\hline $\mathrm{Y} / \mathrm{Nb}$ & 0.31 & 0.32 & 0.31 & 0.3 & 0.33 & 0.33 & 1.08 & 0.89 & 0.89 & 0.79 \\
\hline
\end{tabular}




\section{Continued}

\begin{tabular}{|c|c|c|c|c|c|c|c|c|c|c|}
\hline S. No. & RB11 & $\mathrm{RB} 12$ & RB13 & RB14 & RB15 & RB16 & RB17 & RB18 & RB19 & $\mathrm{RB} 20$ \\
\hline $\mathrm{SiO}_{2} \mathrm{wt} \%$ & 46.83 & 45.62 & 47.64 & 46.75 & 47.4 & 47.11 & 47.64 & 46.8 & 49.32 & 49.8 \\
\hline $\mathrm{TiO}_{2}$ & 1.5 & 1.87 & 1.64 & 1.71 & 1.68 & 1.87 & 2.02 & 1.95 & 0.51 & 0.7 \\
\hline $\mathrm{Al}_{2} \mathrm{O}_{3}$ & 13.28 & 12.96 & 13.4 & 13.72 & 11.78 & 12.45 & 13.8 & 13.15 & 12.9 & 13.64 \\
\hline $\mathrm{Fe}_{2} \mathrm{O}_{3}$ & 11.79 & 13.54 & 12.6 & 14.38 & 12.84 & 14.75 & 14.47 & 14.89 & 10.7 & 13.3 \\
\hline $\mathrm{MnO}$ & 0.18 & 0.19 & 0.18 & 0.21 & 0.19 & 0.22 & 0.21 & 0.22 & 0.15 & 0.15 \\
\hline $\mathrm{MgO}$ & 8.1 & 8.23 & 7.8 & 7.86 & 7.27 & 11.7 & 6.55 & 7.5 & 7.76 & 6.2 \\
\hline $\mathrm{CaO}$ & 12 & 10.87 & 10.1 & 9.87 & 13.21 & 9.4 & 11.53 & 12.1 & 11.78 & 9.4 \\
\hline $\mathrm{Na}_{2} \mathrm{O}$ & 2.56 & 3.14 & 2.97 & 3.25 & 2.56 & 1.36 & 2.36 & 1.57 & 3.26 & 3.21 \\
\hline $\mathrm{K}_{2} \mathrm{O}$ & 1.66 & 0.56 & 1.56 & 0.53 & 0.62 & 0.62 & 0.71 & 0.62 & 1.9 & 0.7 \\
\hline $\mathrm{P}_{2} \mathrm{O}_{5}$ & 0.62 & 0.45 & 0.49 & 0.38 & 0.64 & 0.21 & 0.32 & 0.53 & 0.48 & 0.42 \\
\hline LOI & 1.36 & 2.14 & 1.16 & 1.43 & 2.15 & 1.12 & 0.75 & 0.32 & 1.79 & 2.21 \\
\hline Sum & 99.88 & 99.57 & 99.54 & 100.09 & 100.34 & 100.81 & 100.36 & 99.65 & 100.6 & 99.73 \\
\hline $\mathrm{Mg} \#$ & 57.6 & 54.6 & 55.1 & 52 & 52.8 & 61.1 & 47.3 & 49.9 & 51.6 & 48 \\
\hline $\mathrm{Na}_{2} \mathrm{O} / \mathrm{K}_{2} \mathrm{O}$ & 1.54 & 5.61 & 1.9 & 6.13 & 4.13 & 2.19 & 3.32 & 2.53 & 1.71 & 4.64 \\
\hline $\mathrm{Al}_{2} \mathrm{O}_{3} / \mathrm{TiO}_{2}$ & 8.85 & 8.18 & 8.17 & 7.33 & 7 & 6.65 & 6.83 & 6.74 & 25.29 & 19.48 \\
\hline Cr ppm & 260 & 310 & 290 & 376 & 324 & 371 & 261 & 306 & 35 & 49 \\
\hline $\mathrm{Co}$ & 39 & 49 & 47 & 67 & 54 & 60 & 53 & 68 & 18 & 46 \\
\hline $\mathrm{Ni}$ & 265 & 294 & 255 & 364 & 320 & 344 & 296 & 357 & 160 & 167 \\
\hline $\mathrm{Cu}$ & 75 & 70 & 63 & 79 & 69 & 63 & 73 & 89 & 14 & 83 \\
\hline $\mathrm{Zn}$ & 97 & 101 & 101 & 114 & 105 & 116 & 112 & 119 & 60 & 101 \\
\hline $\mathrm{Sr}$ & 1249 & 536 & 629 & 531 & 1351 & 525 & 733 & 530 & 389 & 449 \\
\hline $\mathrm{Y}$ & 16 & 16 & 17 & 17 & 16 & 17 & 19 & 18 & 17 & 34 \\
\hline $\mathrm{Nb}$ & 17 & 13 & 23 & 27 & 25 & 23 & 36 & 28 & 26 & 22 \\
\hline $\mathrm{La}$ & 19 & 20 & 23 & 23 & 20 & 25 & 27 & 23 & 16 & 25 \\
\hline $\mathrm{Ce}$ & 15 & 16 & 21 & 20 & 23 & 21 & 28 & 25 & 15 & 50 \\
\hline $\mathrm{Pb}$ & 8 & 11 & 9 & 12 & 10 & 11 & 5 & 11 & 14 & 8 \\
\hline $\mathrm{Ba}$ & 306 & 318 & 206 & 258 & 215 & 617 & 1560 & 302 & 928 & 232 \\
\hline $\mathrm{Zr}$ & 334 & 151 & 138 & 126 & 183 & 151 & 189 & 154 & 160 & 260 \\
\hline $\mathrm{Cr} / \mathrm{Ni}$ & 0.98 & 1.05 & 1.14 & 1.03 & 1.01 & 1.07 & 0.87 & 0.85 & 0.22 & 0.3 \\
\hline $\mathrm{Zr} / \mathrm{Nb}$ & 20.17 & 11.61 & 6 & 4.66 & 7.32 & 6.56 & 5.16 & 5.5 & 6.15 & 11.81 \\
\hline $\mathrm{Zr} / \mathrm{Y}$ & 20.87 & 9.43 & 8.11 & 7.41 & 11.43 & 8.88 & 9.94 & 8.55 & 9.41 & 7.64 \\
\hline $\mathrm{Y} / \mathrm{Nb}$ & 0.94 & 1.23 & 0.74 & 0.63 & 0.64 & 0.74 & 0.53 & 0.64 & 0.65 & 1.54 \\
\hline
\end{tabular}




\section{Continued}

\begin{tabular}{|c|c|c|c|c|c|c|c|c|c|c|}
\hline S. No. & $\mathrm{RB} 21$ & RB22 & RB23 & $\mathrm{RB} 24$ & RB25 & RB26 & $\mathrm{RB} 27$ & RB28 & RB29 & RB30 \\
\hline $\mathrm{SiO}_{2} \mathrm{wt} \%$ & 49.87 & 45.4 & 45.74 & 44.67 & 43.13 & 43.96 & 41.79 & 45.85 & 46.07 & 45.1 \\
\hline $\mathrm{TiO}_{2}$ & 0.44 & 0.33 & 0.27 & 0.26 & 0.51 & 1.63 & 0.41 & 0.27 & 1.8 & 2 \\
\hline $\mathrm{Al}_{2} \mathrm{O}_{3}$ & 13.6 & 13.6 & 13.4 & 13.88 & 14.9 & 13.95 & 13.87 & 14.6 & 14.71 & 13.36 \\
\hline $\mathrm{Fe}_{2} \mathrm{O}_{3}$ & 14.78 & 12.9 & 13.6 & 13.98 & 15.2 & 13.95 & 14.57 & 13.41 & 13.25 & 14.22 \\
\hline $\mathrm{MnO}$ & 0.17 & 0.2 & 0.2 & 0.21 & 0.2 & 0.22 & 0.22 & 0.21 & 0.24 & 0.28 \\
\hline $\mathrm{MgO}$ & 5.4 & 7.7 & 8.1 & 7.86 & 8.12 & 8.1 & 8.98 & 8.73 & 7.9 & 8 \\
\hline $\mathrm{CaO}$ & 8.78 & 12.8 & 12.8 & 12.54 & 11.65 & 11.6 & 12.3 & 10.9 & 9.85 & 10.2 \\
\hline $\mathrm{Na}_{2} \mathrm{O}$ & 3.85 & 3.62 & 2.62 & 3.32 & 2.87 & 3.42 & 2.76 & 3.21 & 2.76 & 3.52 \\
\hline $\mathrm{K}_{2} \mathrm{O}$ & 0.88 & 0.93 & 0.53 & 0.65 & 0.74 & 0.62 & 1.95 & 0.6 & 0.7 & 0.82 \\
\hline $\mathrm{P}_{2} \mathrm{O}_{5}$ & 0.37 & 0.26 & 0.42 & 0.39 & 0.43 & 0.51 & 0.56 & 0.49 & 0.38 & 0.36 \\
\hline LOI & 1.82 & 1.65 & 2.12 & 1.65 & 1.78 & 1.74 & 2.13 & 1.75 & 1.85 & 1.76 \\
\hline Sum & 99.96 & 99.39 & 99.8 & 99.41 & 99.53 & 99.7 & 99.54 & 100.02 & 99.51 & 99.62 \\
\hline $\mathrm{Mg} \#$ & 42 & 54.2 & 54.1 & 52.7 & 51.4 & 53.5 & 55 & 56.3 & 54.2 & 52.7 \\
\hline $\mathrm{Na}_{2} \mathrm{O} / \mathrm{K}_{2} \mathrm{O}$ & 4.37 & 3.89 & 4.94 & 5.11 & 3.88 & 5.51 & 1.42 & 5.35 & 3.94 & 4.3 \\
\hline $\mathrm{Al}_{2} \mathrm{O}_{3} / \mathrm{TiO}_{2}$ & 30.9 & 41.21 & 49.63 & 53.38 & 29.21 & 8.55 & 33.82 & 54 & 8.17 & 6.68 \\
\hline Cr ppm & 18 & 92 & 89 & 74 & 299 & 309 & 173 & 101 & 297 & 260 \\
\hline $\mathrm{Co}$ & 68 & 40 & 68 & 72 & 49 & 46 & 59 & 48 & 74 & 67 \\
\hline $\mathrm{Ni}$ & 187 & 250 & 280 & 170 & 232 & 221 & 252 & 238 & 296 & 234 \\
\hline $\mathrm{Cu}$ & 118 & 60 & 65 & 68 & 66 & 76 & 68 & 78 & 71 & 94 \\
\hline $\mathrm{Zn}$ & 124 & 105 & 110 & 114 & 101 & 104 & 104 & 103 & 121 & 126 \\
\hline $\mathrm{Sr}$ & 447 & 799 & 661 & 520 & 843 & 677 & 897 & 721 & 657 & 593 \\
\hline $\mathrm{Y}$ & 60 & 18 & 16 & 14 & 15 & 16 & 17 & 17 & 20 & 21 \\
\hline $\mathrm{Nb}$ & 21 & 31 & 22 & 8 & 20 & 15 & 26 & 14 & 23 & 27 \\
\hline $\mathrm{La}$ & 49 & 23 & 18 & 17 & 16 & 15 & 22 & 14 & 26 & 25 \\
\hline $\mathrm{Ce}$ & 110 & 23 & 14 & 12 & 17 & 15 & 32 & 11 & 44 & 48 \\
\hline $\mathrm{Pb}$ & 15 & 16 & 10 & 13 & 10 & 11 & 5 & 4 & 14 & 21 \\
\hline $\mathrm{Ba}$ & 285 & 1547 & 1953 & 2055 & 767 & 350 & 496 & 378 & 239 & 1022 \\
\hline $\mathrm{Zr}$ & 261 & 109 & 98 & 80 & 203 & 137 & 160 & 118 & 161 & 172 \\
\hline $\mathrm{Cr} / \mathrm{Ni}$ & 0.1 & 0.36 & 0.32 & 0.43 & 1.28 & 1.39 & 0.68 & 0.42 & 1 & 1.1 \\
\hline $\mathrm{Zr} / \mathrm{Nb}$ & 12.42 & 3.51 & 4.45 & 10 & 10.15 & 9.13 & 7.3 & 8.43 & 5.26 & 6.37 \\
\hline $\mathrm{Zr} / \mathrm{Y}$ & 4.35 & 6.05 & 6.12 & 5.71 & 13.53 & 8.56 & 3.53 & 6.94 & 8.05 & 8.19 \\
\hline $\mathrm{Y} / \mathrm{Nb}$ & 2.85 & 0.58 & 0.72 & 1.75 & 0.75 & 1.06 & 0.65 & 1.21 & 0.87 & 0.78 \\
\hline
\end{tabular}




\section{Continued}

\begin{tabular}{|c|c|c|c|c|c|c|c|c|c|c|}
\hline S. No. & RB1 & RB2 & RB3 & RB4 & RB5 & RB6 & RB7 & RB8 & RB9 & RB10 \\
\hline $\mathrm{Pl}$ & 51.8 & 46.36 & 29.36 & 48.67 & 49.18 & 51.48 & 54.35 & 50.32 & 51.33 & 45.47 \\
\hline An & 15.67 & 16.91 & 14.71 & 17.59 & 15.59 & 17.32 & 17.53 & 19.9 & 23.73 & 18.83 \\
\hline $\mathrm{Al}$ & 29.29 & 23.52 & 10.57 & 24.62 & 26.99 & 27.75 & 30.35 & 24.54 & 21.24 & 21.1 \\
\hline Or & 5.85 & 7.15 & 5.85 & 4.85 & 6.44 & 5.43 & 3.9 & 9.75 & 3.78 & 5.2 \\
\hline Di & 18.07 & 18.82 & 48.68 & 18.1 & 19.64 & 17.1 & 24.58 & 15.15 & 10.56 & 30.92 \\
\hline $\mathrm{Ol}$ & 12.02 & 7.17 & - & 8.14 & 13.43 & 9.21 & 9.19 & 7.33 & - & 4.65 \\
\hline $\mathrm{Pe}$ & 4.43 & - & 3.27 & - & 3.52 & - & 1.9 & - & - & 2.14 \\
\hline Ap & 0.57 & 0.65 & 0.76 & 0.97 & 1.23 & 1.12 & 0.93 & 1.25 & 1.72 & 0.74 \\
\hline $\mathrm{Ch}$ & 0.07 & 0.08 & 0.06 & 0.07 & 0.1 & 0.06 & 0.04 & 0.06 & 0.06 & 0.06 \\
\hline Il & 0.5 & 0.51 & 0.41 & 0.46 & 0.46 & 0.47 & 0.4 & 0.45 & 0.45 & 0.38 \\
\hline $\mathrm{He}$ & 13.41 & 12.18 & 10.59 & 12.93 & 12.93 & 12.02 & 11.07 & 13.14 & 13.19 & 11.97 \\
\hline S. No. & RB11 & RB12 & RB13 & RB14 & RB15 & RB16 & RB17 & RB18 & RB19 & RB20 \\
\hline $\mathrm{Pl}$ & 47.67 & 54.42 & 50.35 & 56.76 & 47.46 & 43.43 & 51.41 & 46.45 & 47.82 & 55.31 \\
\hline An & 20.15 & 20.15 & 18.91 & 21.6 & 19.17 & 26.15 & 25.1 & 27.2 & 15.22 & 21.28 \\
\hline $\mathrm{Al}$ & 22 & 27.25 & 25.55 & 27.93 & 22.1 & 11.51 & 20.1 & 13.37 & 28.17 & 27.84 \\
\hline Or & 9.93 & 3.37 & 9.4 & 3.2 & 3.72 & 3.66 & 4.2 & 3.66 & 11.46 & 4.25 \\
\hline Di & 24.96 & 20.7 & 18.62 & 16.1 & 30.1 & 10.84 & 19.21 & 18.79 & 15.67 & 17.14 \\
\hline $\mathrm{Ol}$ & 6.24 & 5.66 & 3.52 & 1.88 & - & - & - & - & - & - \\
\hline $\mathrm{Pe}$ & 0.62 & - & - & - & - & - & - & - & - & - \\
\hline Ap & 1.46 & 1.1 & 1.16 & 0.91 & 1.51 & 0.5 & 0.74 & 0.06 & 1.13 & 0.99 \\
\hline $\mathrm{Ch}$ & 0.06 & 0.07 & 0.06 & 0.08 & 0.07 & 0.07 & 0.06 & 0.53 & 0.02 & 0.02 \\
\hline Il & 0.41 & 0.46 & 0.41 & 0.5 & 0.44 & 0.51 & 0.49 & 0.53 & 0.31 & 0.33 \\
\hline $\mathrm{He}$ & 11.97 & 13.9 & 12.81 & 14.62 & 13.1 & 14.8 & 14.53 & 14.98 & 10.92 & 13.64 \\
\hline S. No. & RB21 & RB22 & RB23 & RB24 & RB25 & RB26 & RB27 & RB28 & RB29 & RB30 \\
\hline $\mathrm{Pl}$ & 57.4 & 38.91 & 53.2 & 45.3 & 50.78 & 51.37 & 26.83 & 56.68 & 56.99 & 55.41 \\
\hline An & 17.56 & 18.54 & 23.81 & 21.54 & 26.14 & 21.33 & 20.25 & 24.19 & 26.26 & 18.61 \\
\hline $\mathrm{Al}$ & 33.17 & 15.7 & 22.68 & 18.15 & 18.37 & 23.53 & 3.53 & 25.94 & 23.95 & 29.7 \\
\hline Or & 5.32 & 5.61 & 3.2 & 3.9 & 4.5 & 3.73 & 11.81 & 3.6 & 4.25 & 4.96 \\
\hline Di & 18.43 & 43.97 & 30.3 & 31.12 & 23 & 22.88 & 30.04 & 21.77 & 12.58 & 19.56 \\
\hline $\mathrm{Ol}$ & - & 2.4 & 4.44 & 3.92 & 7.03 & 7 & 6.34 & 8.43 & 0.27 & 7.9 \\
\hline $\mathrm{Pe}$ & - & 0.15 & - & 0.05 & 0.48 & 2.4 & 0.25 & 0.01 & & 2.9 \\
\hline Ap & 0.88 & 0.63 & 0.99 & 0.93 & 1.02 & 1.2 & 1.32 & 1.16 & 0.9 & 0.86 \\
\hline $\mathrm{Ch}$ & & 0.02 & 0.01 & 0.01 & 0.06 & 0.07 & 0.04 & 0.01 & 0.06 & 0.06 \\
\hline Il & 0.38 & 0.28 & 0.5 & 0.46 & 0.44 & 0.48 & 0.52 & 0.5 & 0.57 & 0.7 \\
\hline $\mathrm{He}$ & 15.06 & 13.2 & 13.92 & 14.3 & 15.55 & 14.24 & 14.96 & 13.65 & 13.57 & 14.53 \\
\hline An & \multicolumn{2}{|c|}{ Anorthite } & \multicolumn{2}{|c|}{ Or } & \multicolumn{2}{|c|}{ Orthoclase } & \multicolumn{2}{|c|}{$\mathrm{Ol}$} & \multicolumn{2}{|c|}{ Olivine } \\
\hline $\mathrm{Al}$ & \multicolumn{2}{|c|}{ Albite } & \multicolumn{2}{|c|}{$\mathrm{Di}$} & \multicolumn{2}{|c|}{ Diopside } & \multicolumn{2}{|c|}{$\mathrm{Pe}$} & \multicolumn{2}{|c|}{ Perovskite } \\
\hline Ap & \multicolumn{2}{|c|}{ Apatite } & \multicolumn{2}{|c|}{ Il } & \multicolumn{2}{|c|}{ Ilmenite } & \multicolumn{2}{|c|}{$\mathrm{Pl}$} & \multicolumn{2}{|c|}{ Plagioclase } \\
\hline $\mathrm{Ch}$ & \multicolumn{2}{|c|}{ Chromite } & \multicolumn{2}{|c|}{$\mathrm{He}$} & \multicolumn{2}{|c|}{ Hematite } & & & & \\
\hline
\end{tabular}


$\mathrm{mm}$ in size. The normative RB indicating to th Hematite content was average $13.40 \mathrm{vol} \%$. Magnetite occurs a phenocrysts scattered through the rock and inclusion within plagioclase, pyroxene and olivine crystals (Figures 2(a)-2(d)). The optical properties of magnetite were black color within ppl and xpl optics. The main secondary minerals occurred as a result of ultration process such as hydrothermal solution, these mineral are iddingsite, calcite and clay.

\subsection{Geochemistry}

\subsubsection{Major Oxides}

The results of major and trace element chemistry of RB volcanic rocks is listed in Table 1 . The RB rocks exhibit a narrow range of silica $\left(\mathrm{SiO}_{2}\right)$ saturation between (41.79 to $\left.54.55 \mathrm{wt} \%\right)$ with an average of $(46.52 \mathrm{wt} \%)$, which is within the average value reported for alkali basalt and basanite by many authors [11] [19] [28]-[32], and it can be classified as basalt using the Total Alkalis-Silica classification scheme [33] (Figure 3). The $\mathrm{Al}_{2} \mathrm{O}_{3}$ contents in the RB samples vary from 9.60 to $14.90 \mathrm{wt} \%$, meanwhile $\mathrm{CaO}$ varies between 7.80 and $17.8 \mathrm{wt} \%$. The binary plot of $\mathrm{SiO}_{2}$ versus $\mathrm{Al}_{2} \mathrm{O}_{3}$ and $\mathrm{CaO}$ exhibits the inverse relationships between both oxides and $\mathrm{SiO}_{2}$ (Figure 4).

The $\mathrm{MgO}$ content of the RB ranged from $5.4 \mathrm{wt} \%$ to $11.7 \mathrm{wt} \%$ with an average of $8.5 \mathrm{wt} \%$. The Mg number $(\mathrm{Mg} \neq)$, defined as the molecular proportion of $(100 \mathrm{MgO} /[\mathrm{MgO}+$ Total Fe] $)$ [30]. It is usually used as a petrogenetic indicator for magma fractionation and its primitive volcanic rocks [34]. The RB exhibited a high $\mathrm{Mg} \neq$, ranging between 42 and 63.8 , with an average of 55.72. The Mg\# of the RB indicates evolved to moderately basalt. As shown in Figure 5, $\mathrm{Mg} \#$ decrease with increasing $\mathrm{SiO}_{2}$. This general trend suggests that fractional crystallization probably plays a role in decreasing $\mathrm{Mg}$-number as a function of increasing $\mathrm{SiO}_{2}$ [35]. The $\mathrm{Mg} \neq$ calculation considers the Fe content in the rocks. It has been reported that values of $\mathrm{Mg} \neq>70$ can be considered as a threshold that characterizes primitive magmas [36]. According to [37] it has been suggested that a $\mathrm{Mg} \neq$ of 65 is a distinct value. Moreover, the $\mathrm{Fe}_{2} \mathrm{O}_{3}$ content of the $\mathrm{RB}$ ranged between $10.4 \mathrm{wt} \%$ and $15.2 \mathrm{wt} \%$, with an average of $13.12 \mathrm{wt} \%$, indicating that the rocks were enriched in Fe. [11] has suggested that $\mathrm{SiO}_{2}$ under saturated magma had a high FeO content of $>11 \mathrm{wt} \%$ and high $\mathrm{MgO}$ content of $>7$. [34] and [38] reported that rocks with high $\mathrm{Mg \#}(>60)$ exhibit lowest contents of $\mathrm{Nb}$ and $\mathrm{Zr}$ and higher contents of $\mathrm{Cr}$ and $\mathrm{Ni}$. All the present studies for the above mentioned, were explained to indicate that the rocks undergo smaller degree of partial melting at high pressures [39].

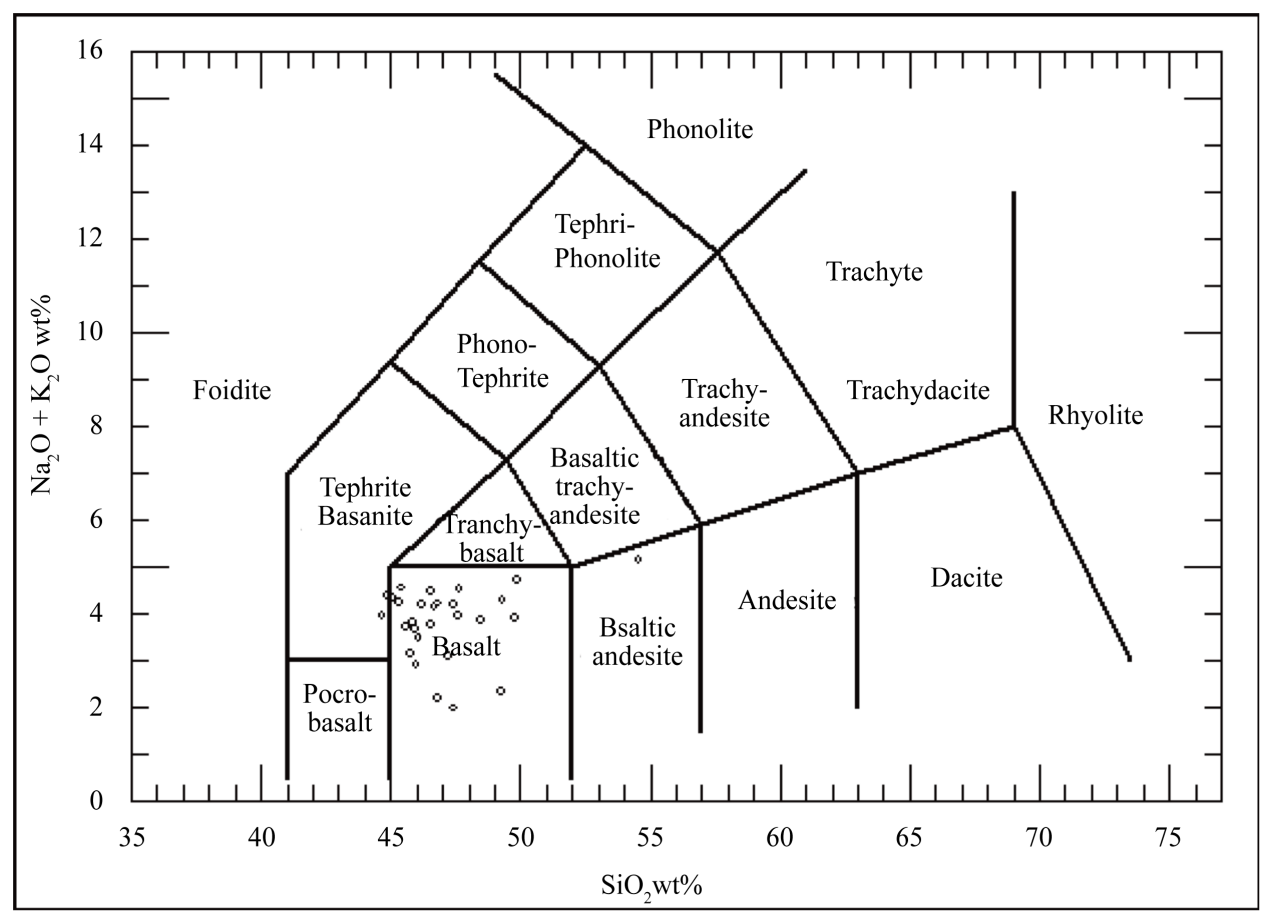

Figure 3. Chemical classification of RB Alkalis vs. Silica [33]. 

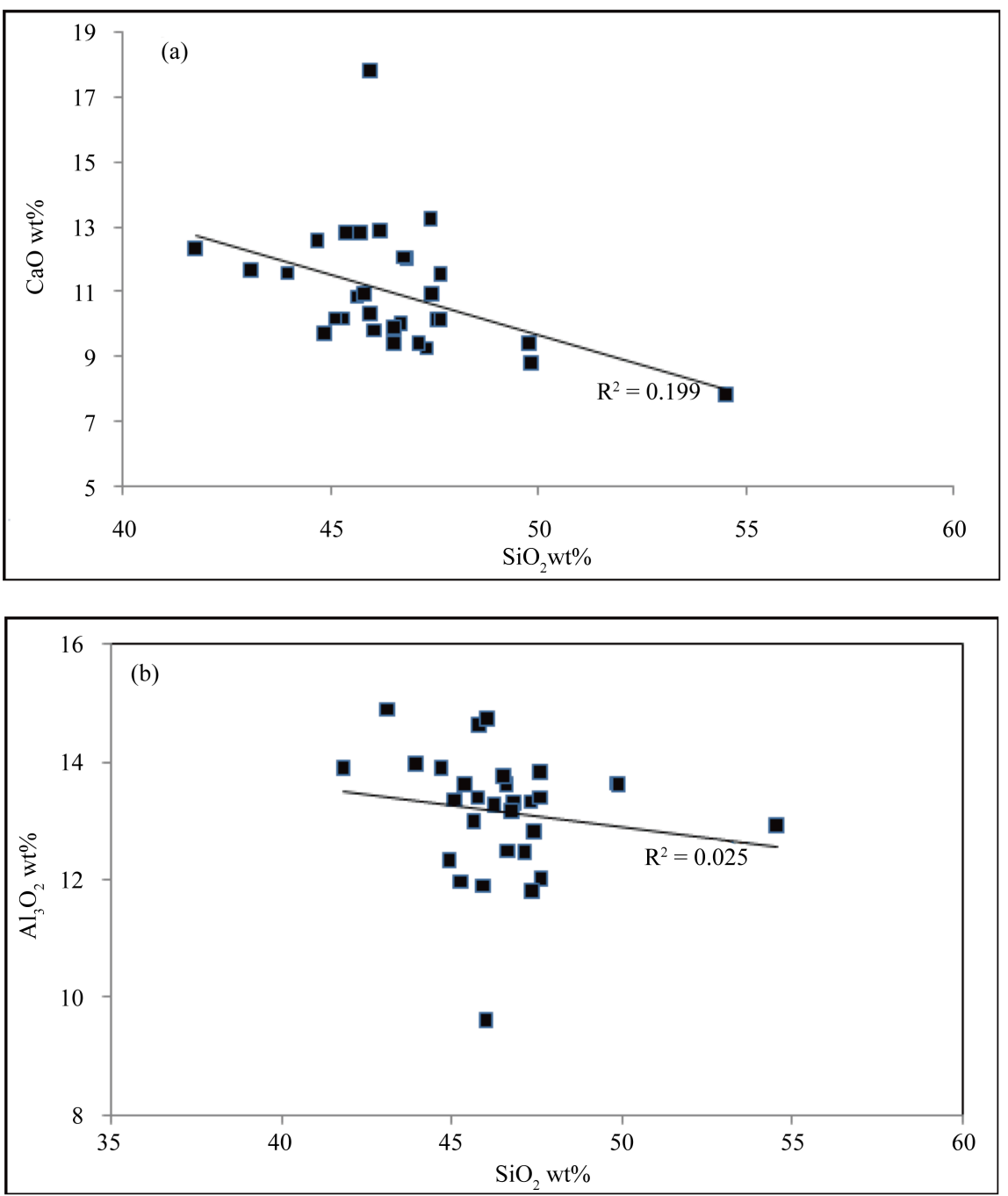

Figure 4. (a) $\mathrm{SiO}_{2} \mathrm{vs} \mathrm{CaO}$ and (b) $\mathrm{SiO}_{2} \mathrm{vs}^{\mathrm{Al}_{2} \mathrm{O}_{3} \text { data. }}$

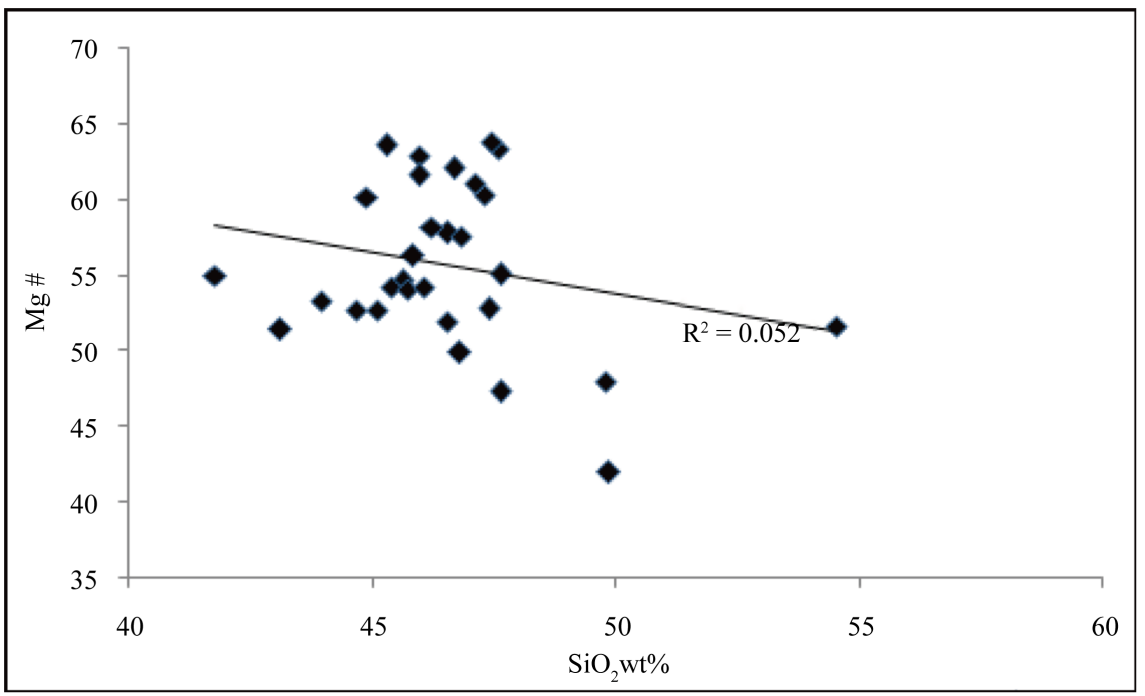

Figure 5. $\mathrm{SiO}_{2} \mathrm{vs} \mathrm{Mg \#} \mathrm{data.}$ 
The RB has been an average $\mathrm{Na}_{2} \mathrm{O}$ and $\mathrm{K}_{2} \mathrm{O}$ content of 2.93 and $0.92 \mathrm{wt} \%$, respectively. The total $\mathrm{Na}_{2} \mathrm{O}+$ $\mathrm{K}_{2} \mathrm{O}$ values were variable in all the samples, exhibiting an average value of $3.84 \mathrm{wt} \%$. The average ratio of $\mathrm{Na}_{2} \mathrm{O} / \mathrm{K}_{2} \mathrm{O}$ was 3.63, and $\mathrm{Na}_{2} \mathrm{O}$ vs. $\mathrm{K}_{2} \mathrm{O}$ indicating the sodic affinity of the rocks [40] (Figure 6), and that of $\mathrm{Al}_{2} \mathrm{O}_{3} / \mathrm{TiO}_{2}$ was 16.12 which suggested the basic affinity of the rock.

\subsubsection{Trace Elements}

The RB was found to have a high content of $\mathrm{Cr}$ and Ni (Table 1). The $\mathrm{Cr}$ content varied between 18 to $385 \mathrm{ppm}$, with an average value $246.87 \mathrm{ppm}$, and $\mathrm{Cr} / \mathrm{Ni}$ ratio of 0.10 to 1.39 . The Ni content ranges between 160 and 364 ppm with average $285.80 \mathrm{ppm}$, suggesting that RB fractionation by presence of olivine and clinopyroxene [41]. The high content of $\mathrm{Cr}$ and $\mathrm{Ni}$ indicated that the parental magma had been derived through partial melting of peridotite mantle source [11] [36]. The binary diagram (Figure 7) shows the Mg\# versus $\mathrm{Cr}$ and $\mathrm{Ni}$. The general trend is decrease of $\mathrm{Cr}$ and $\mathrm{Ni}$ with increasing $\mathrm{Mg} \#$, this result documented with Mudawwara-Quwayra Basaltic Dike [42]. This is most probably resulted from crustal mixing and assimilation of the magma with the country rock which resulted to dilution of $\mathrm{Cr}$ and $\mathrm{Ni}$, where $\mathrm{Cr}$ is related to clinopyroxene [36].

The $\mathrm{Sr}$ and $\mathrm{Zr}$ in the RB were relatively high contents, Sr ranging between 389 to $1351 \mathrm{ppm}$ with average $706.4 \mathrm{ppm}$, and $\mathrm{Zr}$ content ( 80 to $334 \mathrm{ppm}$ ) with average $188 \mathrm{ppm}$ (Table 1). The Reaer Earth Elements (REE) includes $\mathrm{Nb}, \mathrm{Y}, \mathrm{La}$ and Ce content range between ( 8 to $63 \mathrm{ppm}, 13$ to $60 \mathrm{ppm}, 13$ to $49 \mathrm{ppm}$ ), and (11 to 110 $\mathrm{ppm}$ ) respectively (Table 1 ). The average $\mathrm{Zr} / \mathrm{Nb}$ ratio $7.64, \mathrm{Y} / \mathrm{Nb}$ ratio 0.84 and $\mathrm{Zr} / \mathrm{Y}$ ration 10.29 . These ratio decomented by [43] reported for the intercontinental alkali basalt.

\section{Discussion}

The major and trace elements of the RB were used to construct discriminatory diagrams, to help for the classification, nomenclature, and interpretation of the tectonic setting of the RB. Classification for [33], the RB samples plotted in the alkaline to subalkaline rock field (Figure 8(a)). The AFM diagram shows the RB samples plotted in the calcalkaline series (Figure 8(b)). The ternary diagram for Ti-Zr-Y based on [44] was distinguished among island-arc tholeiitas, MORB, calc-alkaline, and intraplate basalt, all the RB samples were plotted within the plate basalt field (Figure 9(a)). On the other hand, Ti-Zr-Sr digram shows all the RB samples plotted in the calcalkaline basalt field (Figure 9(b)) [44]. The $\mathrm{MgO}^{-} \mathrm{FeO}_{(\text {tot) }}-\mathrm{Al}_{2} \mathrm{O}_{3}$ diagram for [45], shows the RB samples ploted within continental basalt field (Figure 10).

The low content of $\mathrm{SiO}_{2}$ (41.79 to $54.55 \mathrm{wt} \%$ ) and high content of $\mathrm{MgO}$ (5.4 to $11.7 \mathrm{wt} \%$ ) and total $\mathrm{FeO}$ (10.40 - $15.20 \mathrm{wt} \%)$ indicated the natural fractionation of the RB. The high concentration of $\mathrm{Cr}$ (18 to $385 \mathrm{ppm})$ with average $246.87 \mathrm{ppm}$ is consistent with findings reported for primary magma |(e.g., $241 \mathrm{ppm}$ [30], 257.6 ppm [11], $313.6 \mathrm{ppm}$ [19], $125.3 \mathrm{ppm}$ [46], $95 \mathrm{ppm}$ [42]. The high Mg\# (average 55.66) for RB is similar to that reported for rock affected by fractionation or accumulation of clinopyroxene, orthopyroxene, and olivine and

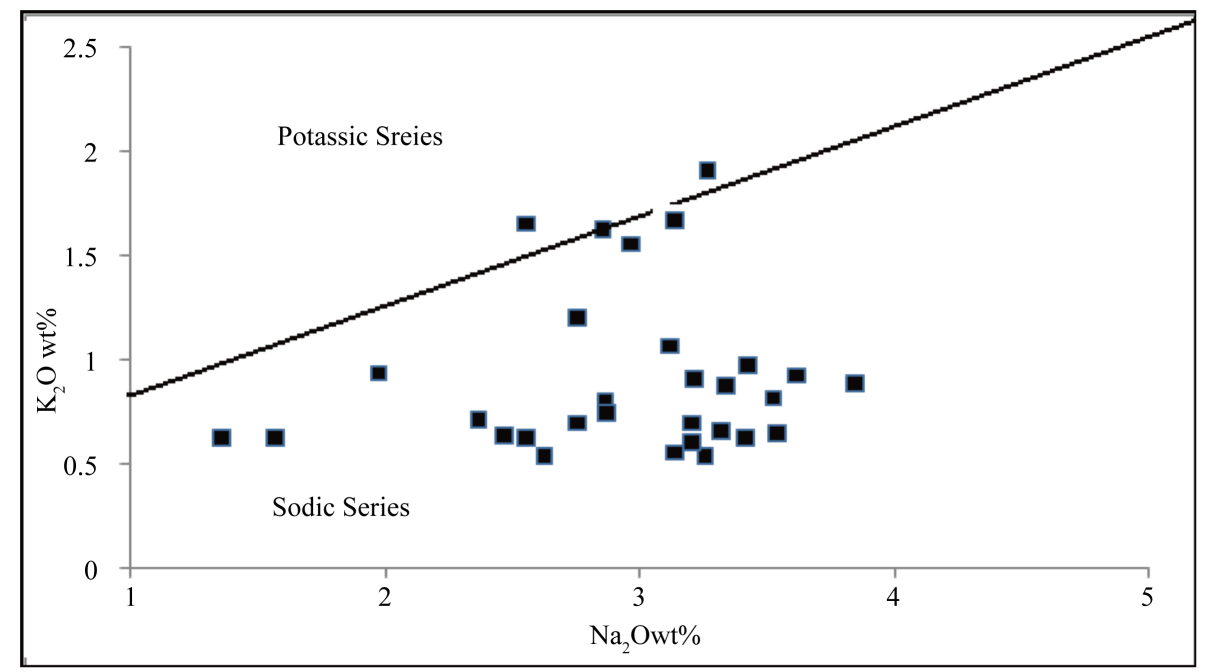

Figure 6. $\mathrm{K}_{2} \mathrm{O}$ vs $\mathrm{Na}_{2} \mathrm{O}$ showing the sodic affinity of the $\mathrm{RB}$ samples [44]. 

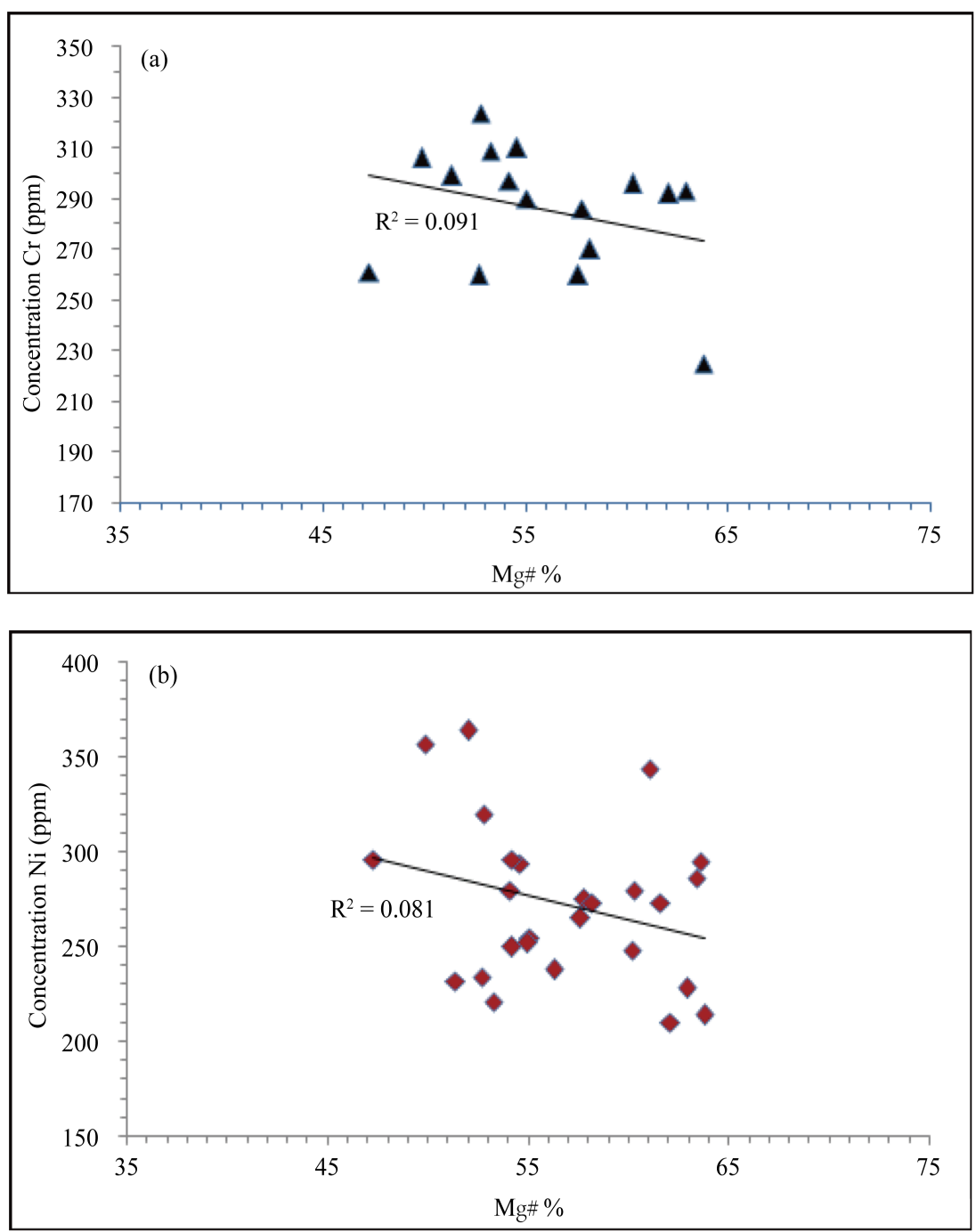

Figure 7. (a) $\mathrm{Mg \#}$ vs $\mathrm{Cr}$ and (b) $\mathrm{Mg \#}$ vs $\mathrm{Ni}$.
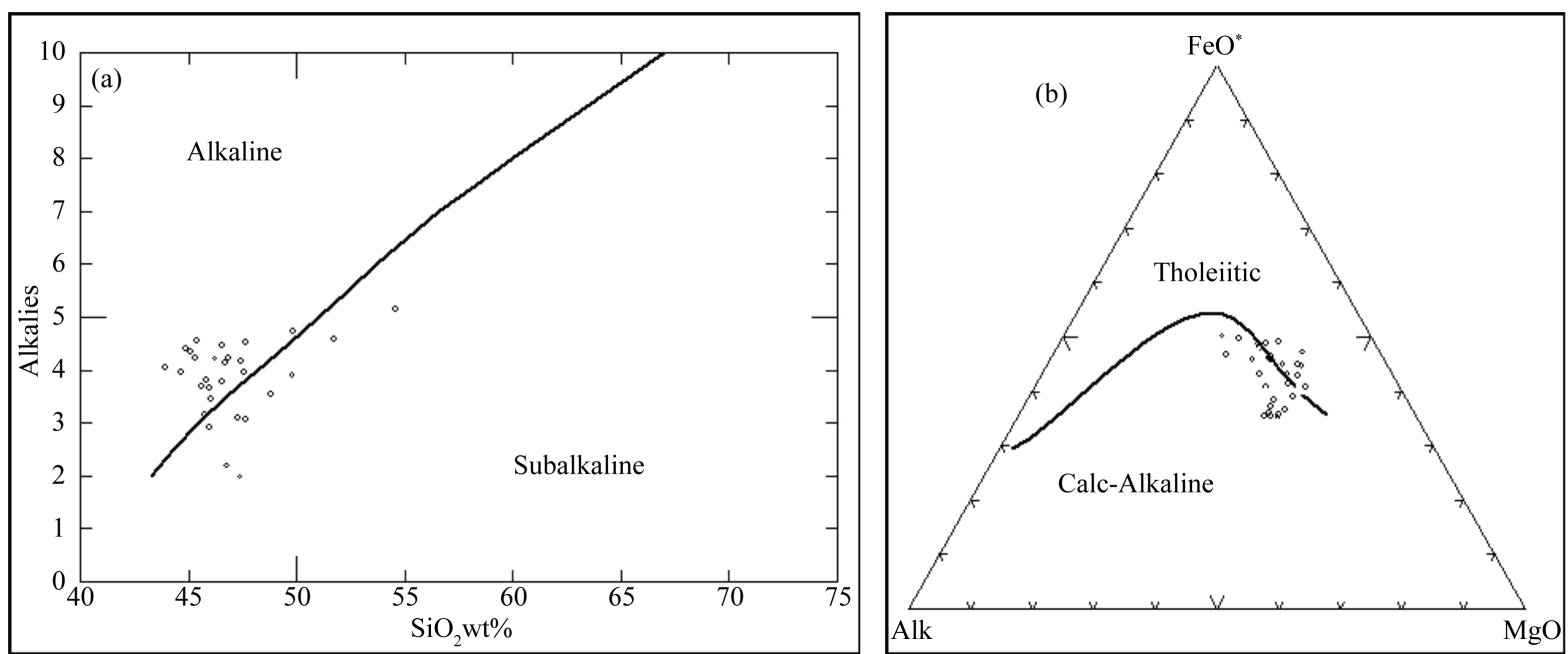

Figure 8. (a) Total alkali vs $\mathrm{SiO}_{2}$ in RB; (b) AFM diagram showing the boundary between the calc-alkaline field and tholeiitic field for RB [33]. 

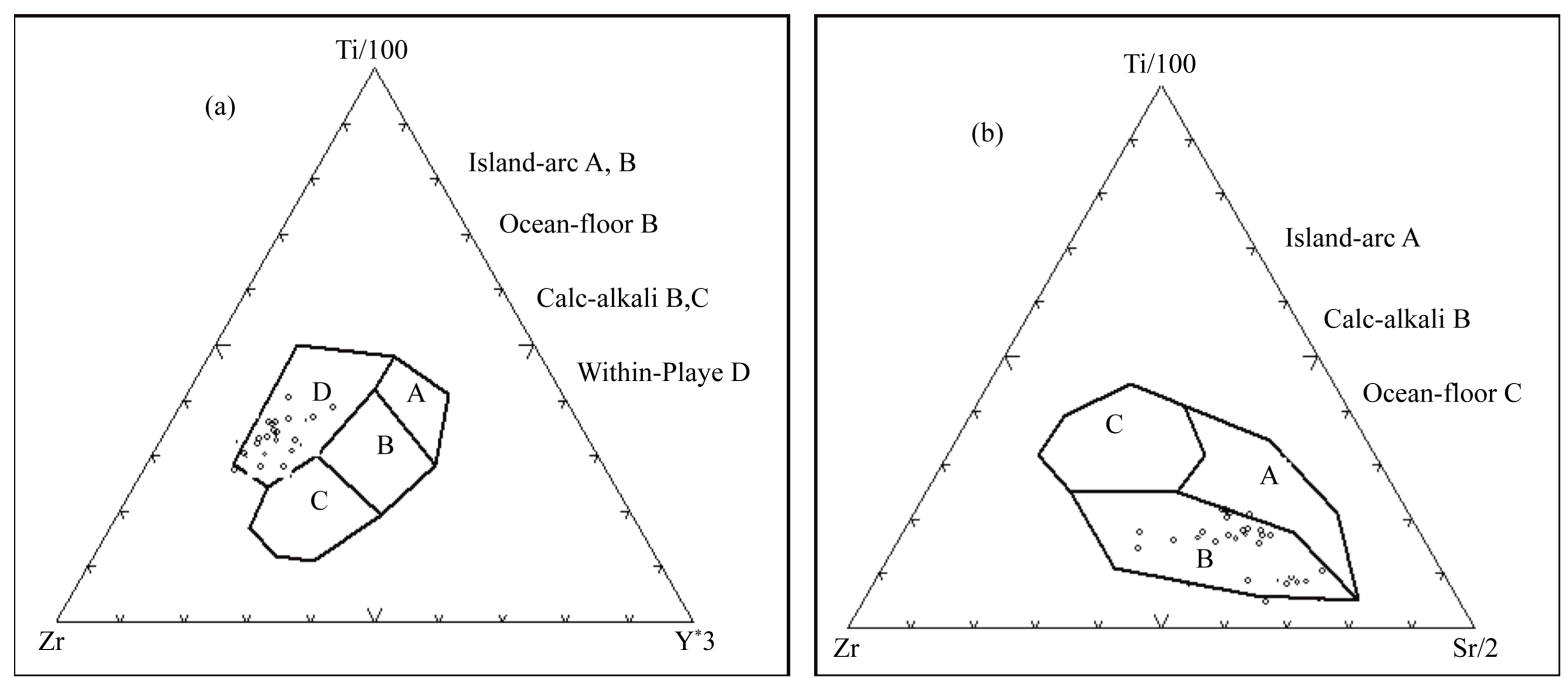

Figure 9. (a) Ti-Zr-Y discrimination diagram for RB [44]; (b) Ti-Zr-Sr discrimination diagram for RB [45].

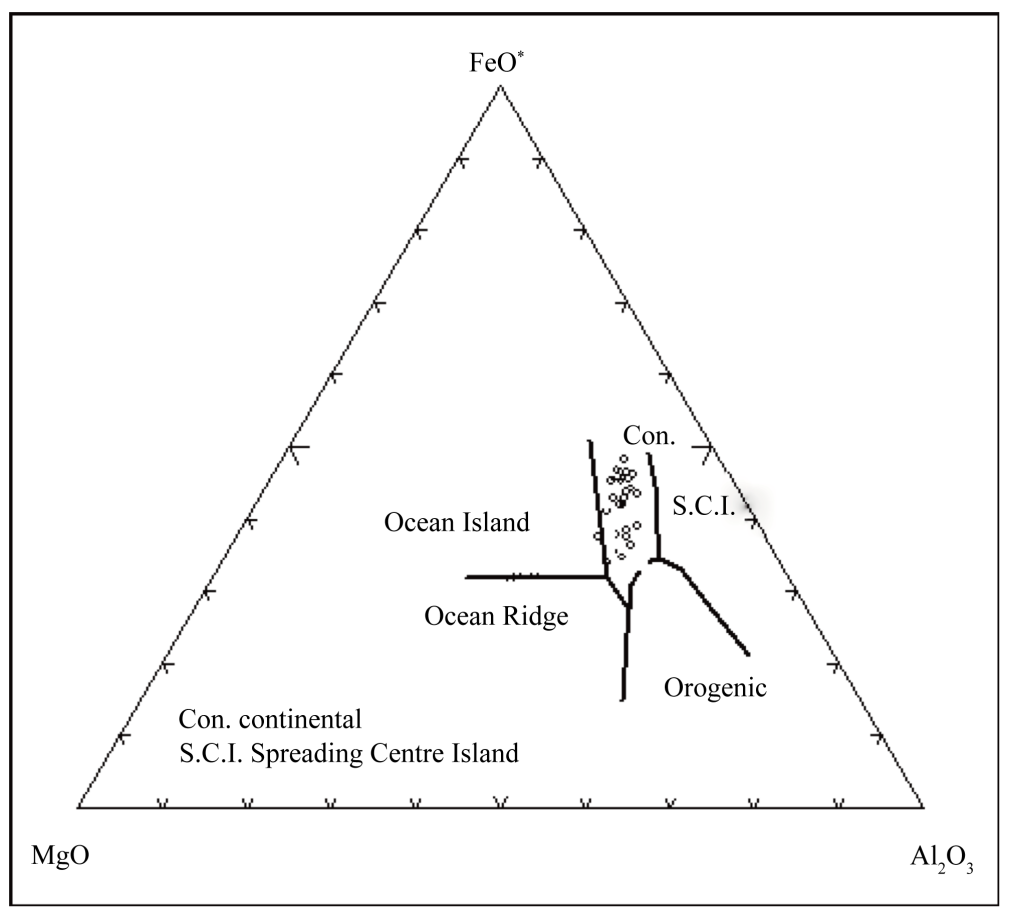

Figure 10. Discrimination digram $\mathrm{MgO}-\mathrm{FeO}_{(\text {tot })}-\mathrm{Al}_{2} \mathrm{O}_{3}$ for $\mathrm{RB}[45]$.

plagioclase [34]. The Rayleigh fractionation equation for [47], are used to modeled the mineral fractionation $C_{l} / C_{o}=F^{(D-1)}$, where $C_{l}$ is the concentration of a trace element in the residual melt; $C_{o}$ is the concentration of a trace element in the original melt; $F$ is the fraction of melt that remains, and $D$ is the bulk partition coefficient. The partition coefficient of $\mathrm{Sr}$ and Ba modeled of the mineral fractionation vector diagram in Figure 11, which indicated that the RB rock samples had fractions of clinopyroxene, orthopyroxene, olivine, and traces of plagioclase.

The batch melting equation for [47] are used to study the degree of partial melting of the RB. The equation $C_{l} / C_{o}=1 /\left[D_{o}+F^{-\left(1-D_{o}\right)}\right.$, where $F$ is the melting degree; $C_{o}$ and $C_{l}$ are the concentrations of the elements in the source (original) and liquid, respectively, and $D_{o}$ is the bulk distribution coefficient of the elements in the initial assemblage and minerals entering into the liquid. The distribution coefficient model for $\mathrm{Cr}$ and $\mathrm{Co}$, and the 


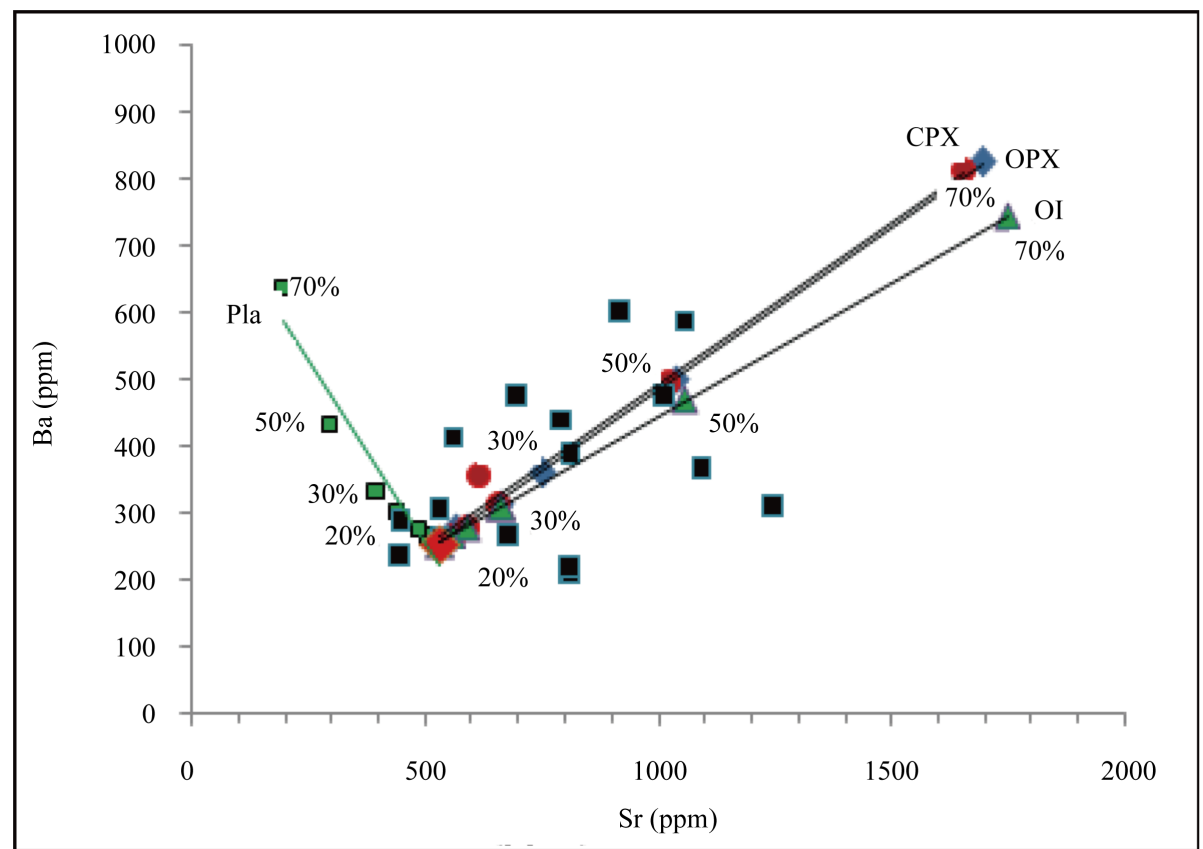

Figure 11. Sr vs Ba modeled mineral fractionation vector diagram for the RB rock samples studied. Fractionation trends are shown for $10 \%, 20 \%, 30 \%, 50 \%$ and $70 \%$, fractional crystallization of minerals: plagioclase (Plag), clinopyroxene (Cpx), orthopyroxene (Opx), and olivine $(\mathrm{Ol})$.

concentration of the large ion lithophile elements (LILE) was found to exhibit primitive composition [27]. The degree of partial melting (F) calculated by using the concentrations of the oceanic crust sources. The studied RB samples shows an average partial melting degree of around 10\%, which documented with the previously published results for Jordanian and Arabian interpolate basalt [11] [19] [31] [48].

The geochemical information for major and trace elements are used to explain the natural source of $\mathrm{RB}$, and it is used to explain the mantle sources of partial melting. This indicates by using trace element ratios, such as $\mathrm{Zr} / \mathrm{Nb}$ and $\mathrm{K} / \mathrm{Ba}$ ratios [49]. The high content of $\mathrm{Zr} / \mathrm{Y}$ and $\mathrm{TiO}_{2} / \mathrm{Y}$ ratios and low content of $\mathrm{Y}$ indicate that to the garnet - bearing source rocks [50] [51]. The spider diagram for normal mid-ocean ridge basalt (NMORB) for the studied volcanic rocks (Figure 12(a)) presented enrichment of the strongly incompatible LILE such as Ba and $\mathrm{K}$, depletion of $\mathrm{Nb}$ relatively to $\mathrm{K}$, enrichment of $\mathrm{Pb}$ over $\mathrm{Ce}$, and light rare earth elements (LREE) enrichment La over heavy rare earth elements (HREE) and Y, which showed high similarity to MORB. The mafic volcanic $\mathrm{RB}$ exhibited negative $\mathrm{Nb}$ anomalies and positive $\mathrm{Pb}$ anomalies, but presented higher LILE enrichments. The negative anomalies of $\mathrm{Ba}, \mathrm{Sr}, \mathrm{P}$, and Ti may be attributed to the fractionation of feldspar for $\mathrm{Ba}$ and $\mathrm{Sr}$ depletion, apatite for $\mathrm{P}$ depletion, and (Fe-Ti) oxides for Ti depletion [38]. The primitive mantle value of the rock (Figure 12(b)) showed a positive $\mathrm{Nb}$ peak, which conforms to the tertiary to recent continental alkali basalt provinces [52]-[54] and indicates that the RB is the product of lithosphere from upwelling asthenospheric mantle [19] [34] [36] [55].

\section{Conclusions}

The RB introduced within Miocene to Pleistocene volcanism at central Jordan. It is produced within intraplate to continental calc-alkaline to alkali basalt. The samples study covered at Al-Rabaa basalt flow about $20 \mathrm{~km}^{2}$. The following is the conclusion of the present study:

1) The mineral composition of RB is as follows: plagioclase, pyroxene, olivine, and opaque minerals (magnetite), with secondary minerals such as iddingzite, calcite, and clay. The main textures observed trachytic, glomeroporphyritic, vesicular, amygdaloidal, ophtic and supohtic.

2) The chemical classification of RB uses major elements into basaltic-calc-alkaline to alkali basalt and includes into sodic series. 

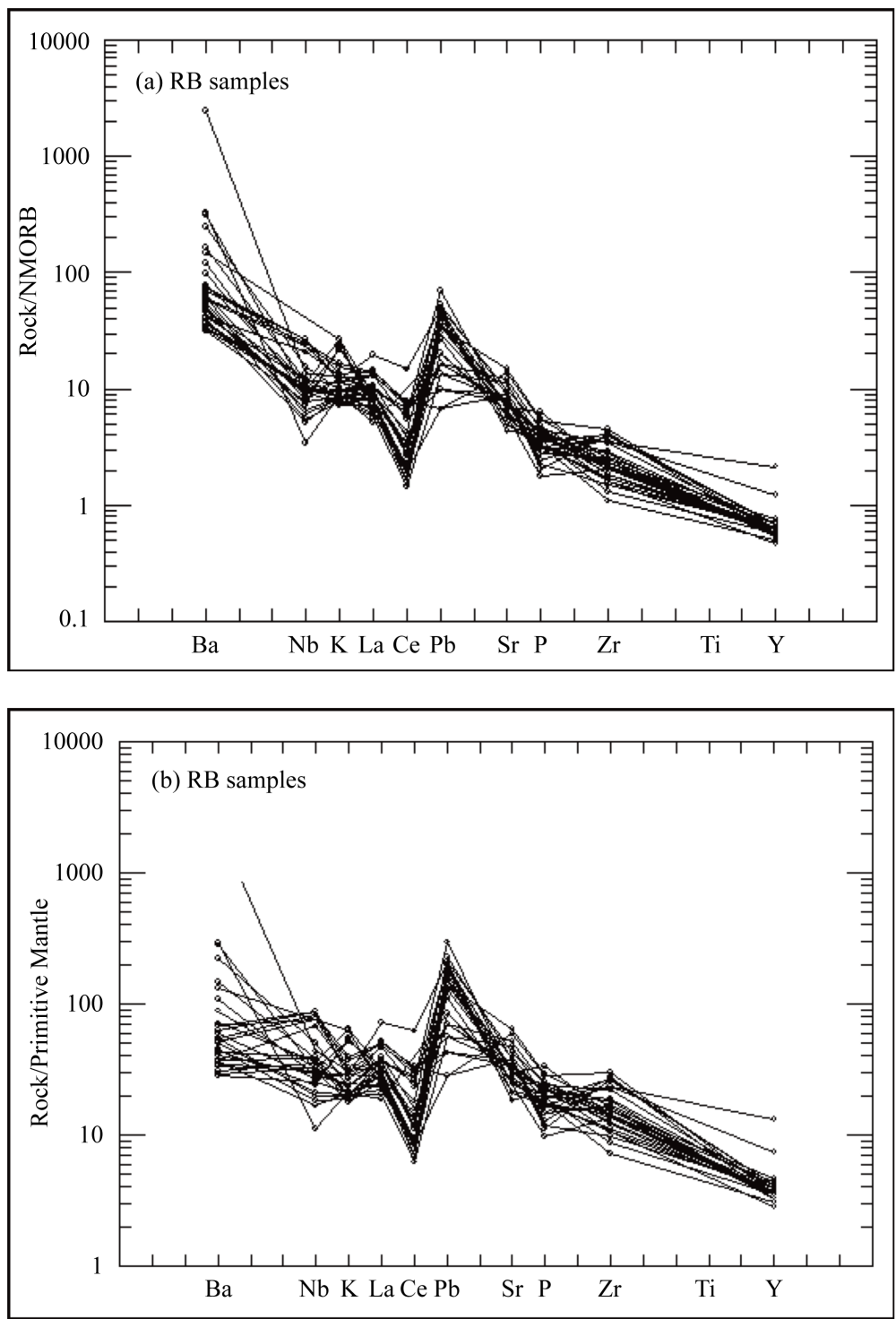

Figure 12. Spider diagram of incompatibility elements from the RB. (a) MORB-Normalized incompatible element plots [43]; (b) Normalized primitive mantle source elements show an increasing incompatibility with mantle rocks [52].

3) The tectonic setting for the discrimination diagram showed that the RB samples were ploted within interplate basalt, calc-alkaline basaltic and continental basaltic field.

4) The vector diagram for fractional crystallization model for RB showed the presence of clinopyroxene, orthoproxene, olivine, and traces of plagioclase. Documented with the published results for Jordanian and Arabian interpolate basalt, the distribution coefficient of $\mathrm{Cr}$ and $\mathrm{Co}$ indicated a partial batch melting of $\mathrm{RB}$ around to $10 \%$.

5) The spider diagram for NMORB and MORB showed enrichment of the incompatible LILE such as Ba and $\mathrm{K}$, and depletion of $\mathrm{Nb}$ relatively to $\mathrm{K}$, enrichment of $\mathrm{Pb}$ over $\mathrm{Ce}$, and Light Rare Earth Elements (LREE) enrichment La over heavy rare earth elements (HREE) and Y. Furthermore, RB exhibited negative Nb and positive $\mathrm{Pb}$ anomalies, which indicated that $\mathrm{RB}$ was a product of lithosphere from upwelling asthenospheric mantle.

\section{Acknowledgements}

The author is thankful to Natural Resources Authority (NRA) for assistance in the analysis by Ione Inductively 
Conductive Coupled Plasma Emission Spectroscopy (ICP-AES) analysis for trace elements (contribution for Regional Geochemical Prospecting Project for central Jordan). The author is also grateful to Al al-Bayt University (Water, Environment and Arid Regions Research Centre), for performing the XRF analysis for major elements, and to Mr. Adnan Mashaqbeh for help in the preparation of thin sections. The authors are also grateful to Engineer Aya Bany Yaseen for her help in the field and Photomicrographs preparation. The author is indebted and grateful to the anonymous reviewers for their comments that helped in improving the manuscript.

\section{References}

[1] Almond, D.C. (1986) The Relation of Mesozoic Magmatic Volcanicity to Tectonics in the Afro-Arabian Dome. Journal of Volcanology and Geothermal Research, 28, 225-246. http://dx.doi.org/10.1016/0377-0273(86)90024-7

[2] Garfunkel, Z. (1989) Tectonic Setting of Phanerozoic Magmatism in Israel. Israel Journal of Earth Sciences, 38, 5174.

[3] Burke, K. (1996) The African Plate. South African Journal of Geology, 99, 341-409.

[4] Al-Malabeh, A., Al-Fugha, H. and El-Hasan, T. (2004) Petrology and Geochemistry of Late Precambrian Magmatic Rocks from Southern Jordan. Neues Jahrbuch für Geologie und Paläontologie, 233, 333-350.

[5] Coleman, R. and McGuire, A. (1988) Magma Systems Related to the Red Sea Opening. Tectonophysics, 150, 77-100. http://dx.doi.org/10.1016/0040-1951(88)90296-X

[6] Camp, V. and Roobol, M. (1992) Upwelling Asthenosphere beneath Western Arabian and Its Regional Implication. Journal of Geophysical Research, 97, 15255-15271. http://dx.doi.org/10.1029/92JB00943

[7] Pick, R., Deniel, C., Coulon, C., Yirgu, G. and Marty, B. (1999) Isotopic and Trace Element Signatures of Ethiopian Flood Basalts: Evidence for Plume-Lithosphere Interactions. Geochimica et Cosmo-Chimica Acta, 63, 2263-2279. http://dx.doi.org/10.1016/S0016-7037(99)00141-6

[8] Baker, J., Menzies, M.A., Thirlwall, M.F. and Macpherson, G.G. (1997) Petrogenesis of Quaternary Interpolate Volcanism, Sana'a Yemen: Implication for Plume Lithosphere Interaction and Polybaric Melt Hybridization. Journal of Petrology, 38, 1359-1390. http://dx.doi.org/10.1093/petroj/38.10.1359

[9] White, R. and Mckenzie, D. (1989) Magmatism at Rift Zones the Generations of Volcanic Continental Margins and Flood Basalts. Journal of Geophysical Research, 94, 7685-7729. http://dx.doi.org/10.1029/JB094iB06p07685

[10] Barberi, F., Capaldi, G., Gasperini, P., Marinelli, G., Santacroce, R., Scandone, R., Treuil, M. and Varet, J. (1979) Recent Basaltic Volcanism of Jordan and Its Implications on the Geodynamic Evolution of the Afro-Arabian Rift System. Academia Nazionale Dei Lincei, Att Del Convegni Lincei, Rome, 667-683.

[11] Shaw, J.E., Baker, J.A., Menzies, M.A., Thirlwall, M.F. and Ibrahim, K.M. (2003) Petrogenesis of the Largest Intraplate Volcanic Field on the Arabian Plate (Jordan): A Mixed Lithosphere-Asthenosphere Source Activated by Lithospheric Extension. Journal of Petrology, 44, 1657-1679. http://dx.doi.org/10.1093/petrology/egg052

[12] Ibrahim, K. and Al-Malabeh, A. (2006) Geochemistry and Volcanic Features of Harrat El-Fahda, a Young Volcanic Field in Northwest Arabia, Jordan. Journal of Asian Sciences, 127, 127-154. http://dx.doi.org/10.1016/j.jseaes.2005.01.009

[13] Basem, K. (1992) Geology of the Ar-Rabba Area. Sheet No.3152IV, Geology Directorate, Natural Resources Authority, Bulletin 22, Amman, Jordan.

[14] Moffat, D. (1988) A Volcano Tectonic Analysis of the Cenozoic Continental Basalts of Northern Jordan: Implications for Hydrocarbon Prospectively in the Block B Area. Unpublished Report, University College of Swansea, Swansea.

[15] Duffield, W., Mckee, E., El-Salem, F. and Feimeh, M. (1988) K-Ar Ages Chemical Composition and Geothermal Significance of Cenozoic Basalt near the Jordan Rift. Geothermics, 17, 635-644. http://dx.doi.org/10.1016/0375-6505(88)90048-X

[16] Ilani, S., Harlavan, Y., Tarawneh, K., Rabba, I., Weinberger, R., Ibrahim, K., Peltz, S. and Steinitz, G. (2001) New K-Ar Ages of Basalts from the Harrat Asham Volcanic Field in Jordan: Implications for the Span and Duration of Upper Mantle Upwelling Beneath the Western Arabian Plate. Geology, 29, 171-174. http://dx.doi.org/10.1130/0091-7613(2001)029<0171:NKAAOB $>2.0 . C O ; 2$

[17] Bender, F. (1974) Geology of the Arabian Peninsula, Jordan. US Geological Survey Professional Paper, 36, $560-561$.

[18] Al-Malabeh, A. (2003) Geochemistry and Volcanology of Jabal Al-Rufiyat, Strombolian Monogenic Volcano, Jordan. Dirasat, 30, 125-140.

[19] El-Hasan, T. and Al-Malabeh, A. (2008) Geochemistry, Mineralogy and Petrogenesis of El-Lajjoun Pleistocene Alkali Basalt of Central Jordan. Jordan Journal of Earth and Environmental Sciences, 1, 53-62.

[20] Steinitz, G. and Bartov, Y. (1992) The Miocene-Pleistocene History of the Dead Sea Segment of the Rift in Light of 
K-Ar Ages of Basalts. Israel Journal of Earth Sciences, 40, 199-208.

[21] Al-Harithi, T. (1982) Stratigraphy and Micropaleontology of Lower Belqa Group. MSc Thesis, Unvercity of Jordan, Amman.

[22] Basha, S.H. and Mikbel, S.R. (1987) A Contribution to the Stratigraphy and Tectonics of the Lower ed-Dhira-East of Lisan (Jordan Rift). Dirasat, 12, 227-246.

[23] Hatcher Jr., R., Zietz, I., Regan, R.D. and Abu-Ajammieh, M. (1981) Sinistra Strike-Slip Motion on the Dead Sea Rift: Confirmation from New Magnetic Data. Geology, 9, 458-462. http://dx.doi.org/10.1130/0091-7613(1981)9<458:SSMOTD>2.0.CO;2

[24] Hollocher, K. (2004) CIPW Norm Calculation Program. Geology Department, Union.

[25] Basem, K. (1988) Geological Map Ar-Rabba Area, Scale 1:50,000. Natural Resources Authority, Geological Directorate, Amman.

[26] Kerr, P. (1977) Optical Mineralogy. John Wiley and Sons, New York.

[27] Cox, K., Bell, J. and Pankhurst, R. (1979) The Interpretation of Igneous Rocks. Springer, London. http://dx.doi.org/10.1007/978-94-017-3373-1

[28] Thorpe, W. and Graham, P. (1993) A Province Study of Jordanian Basalt Vessels of the Chalcolithic and Early Bronze Age 1 Periods. Paléorient, 19, 51-63. http://dx.doi.org/10.3406/paleo.1993.4596

[29] Cebria, J. and Lopez-Ruiz, J. (1995) Alkali Basalt and Leucitites in an Extensional Interpolate Setting: The Late Cenozoic Calatrava Volcanic Province (Central Spain). Lithos, 35, 27-46. http://dx.doi.org/10.1016/0024-4937(94)00027-Y

[30] Watts, B.G., Bennett, M.E., Kopp, O.C. and Mattingly, G.L. (2004) Geochemistry and Petrography of Basalt Grindstones from the Karak Plateau, Central Jordan. Geoarchaeology, 19, 47-69. http://dx.doi.org/10.1002/gea.10103

[31] Shaw, J.E., Baker, J.A., Kent, A.J., Ibrahim, K.M. and Menzies, M.A. (2007) The Geochemistry of the Arabian Lithospheric Mantle-A Source for Intraplate Volcanism. Journal of Petrology, 48, 1495-1512. http://dx.doi.org/10.1093/petrology/egm027

[32] Al-Malabeh, A. (2009) Cryptic Mantle Metasomatism: Evidences from Spinel Lherzolite Xenoliths/Al-Harida Volcano in Harrat Al-Shaam, Jordan. American Journal of Applied Sciences, 6, 2085-2092. http://dx.doi.org/10.3844/ajassp.2009.2085.2092

[33] Le Maitre, R.W., Bateman, P., Dudek, A., Keller, J., Lameyre Le Bas, M.J., Sabine, P.A., Schmid, R., Sorensen, H., Streckeisen, A., Woolley, A.R. and Zanettin, B. (1989) A Classification of Igneous Rocks and Glossary of Terms. Blackwell, Oxford.

[34] Ma, G.S.-K., Malpas, J., Xenophontos, C. and Chan, G.H.-N. (2011) Petrogenesis of Latest Miocene-Quaternary Continental Intraplate Volcanism along the Northern Dead Sea Fault System (Al-Ghab-Homs Volcanic Field), Western Syria: Evidence for Lithosphere-Asthenoshere Interaction. Journal of Petrology, 1-30.

[35] Shaw, J. (2003) Geochemistry of Cenozoic Volcanism and Arabian Lithospheric Mantle in Jordan. Unpublished PhD Thesis, University of London, London.

[36] Wilson, M. (1989) Igneous Petrogenesis. Unwin Hyman Ltd., London, 466. http://dx.doi.org/10.1007/978-1-4020-6788-4

[37] Clague, D. and Ferry, F. (1982) Petrology and Trace Elements Geochemistry of Honolulu Volcanism: Implication for the Ocean Mantle below Hawaii. Journal of Petrology, 23, 447-504. http://dx.doi.org/10.1093/petrology/23.3.447

[38] Moghazi, A.M. (2003) Geochemistry and Petrogenesis of a High-K Calc-Alkaline Dokhan Volcanic Suite, South Safaga Area, Egypt: The Role of Late Neoproterozoic Crustal Extension. Precambrian Research, 125, 161-178. http://dx.doi.org/10.1016/S0301-9268(03)00110-4

[39] Yaseen, I.A.A.B. (2014) Contribution to the Petrography, Geochemistry, and Petrogenesis of Zarqa-Ma'in Pleistocene Alkali Olivine Basalt Flow of Central Jordan, International Journal of Geosciences, 5, 657-672. http://dx.doi.org/10.4236/ijg.2014.56059

[40] Middlemost, E. (1975) The Basalt Clan. Earth-Science Reviews, 11, 337-564. http://dx.doi.org/10.1016/0012-8252(75)90039-2

[41] Winter, J.D. (2001) An Introduction to Igneous and Metamorphic Petrology. Prentice Hall Inc., Upper Saddle River, 697.

[42] Alnawafleh, H., Tarawneh, K., Ibrahim, K., Zghoul, K., Titi, A., Rawashdeh, R., Moumani, K. and Masri, A. (2015) Characterization and Origin of the Miocene Mudawwara-Quwayra Basaltic Dike, Southern Jordan. International Journal of Geosciences, 6, 869-881. http://dx.doi.org/10.4236/ijg.2015.68071

[43] Pearce, J., Harris, N. and Tindle, A. (1984) Trace Element Discrimination Diagram for the Tectonic Interpretation of 
Granitic Rocks. Journal of Petrology, 25, 956-983. http://dx.doi.org/10.1093/petrology/25.4.956

[44] Pearce, J.A. and Cann, J.R. (1973) Tectonic Setting of Basic Volcanic Rocks Determined Using Trace Element Analyses. Earth and Planetary Science Letters, 19, 290-300. http://dx.doi.org/10.1016/0012-821X(73)90129-5

[45] Pearce T.H., Gorman, B.E. and Birkett, T.C. (1977) The Relationship between Major Element Chemistry and Tectonic Environment of Basic and Intermediate Volcanic Rocks. Earth and Planetary Science Letters, 36, 121-132. http://dx.doi.org/10.1016/0012-821X(77)90193-5

[46] Al-Fugha, H. and Al-Amaireh, M. (2007) Petrology and Origin of Ultramafic Xenoliths from Northeastern Jordan Volcanoes. American Journal of Applied Sciences, 4, 491-495. http://dx.doi.org/10.3844/ajassp.2007.491.495

[47] Rollinson, H.R. (1993) Using Geochemical Data: Evaluation, Presentation, Interpretation. Longman Scientific and Technical, England.

[48] Al-Malabeh, A., El-Hasan, T., Lataifeh, M. and Shea, M. (2002) Geochemical and Mineralogical Related Magnetic Characteristics of the Tertiary-Quaternary (Umm Al-Qutein) Basaltic Flows from the Basaltic Field of Harrat El-Jabban Northeast Jordan. Physica B: Condensed Matter, 32, 396-403. http://dx.doi.org/10.1016/S0921-4526(02)01083-9

[49] Peltz, S. and Bratosia W. (1986) New Data on the Geochemistry of the Quaternary Basalts in Pensani Mountains. Geophics, 71, 389-403.

[50] Frey, F.A., Green, D.H. and Roy, S.D. (1978) Integrated Models of Basalt Petrogenesis: A Study of Quartz Theoliites to Olivine Melilites from South Eastern Australia Utilizing Geochemical and Experimental Petrological Data. Journal of Petrology, 19, 463-513. http://dx.doi.org/10.1093/petrology/19.3.463

[51] Jenner, G., Gawood, P., Rautenschlein, M. and White, W. (1987) Composition of Back-Arc Basin Volcanic Valufa Ridge Lau Basin: Evidence for a Slab-Derived Component in Their Mantle Source. Journal of Volcanology and Geothermal Research, 32, 209-222. http://dx.doi.org/10.1016/0377-0273(87)90045-X

[52] Sun, S.S. and MacDonough, W.F. (1989) Chemical and Isotopic Systematic of Oceanic Basalts Implications for Mantle Composition and Processes in Magmatism in the Ocean Basins. Geological Society, London, Special Publication, 42, 313-345. http://dx.doi.org/10.1144/GSL.SP.1989.042.01.19

[53] Norry, M.J. and Fitton, J.G. (1993) Compositional Differences between Oceanic and Continental Basic Lavas and Their Significance. In: Hawkesworth, C.J. and Norry, M.J., Eds., Continental Basalts and Mantle Xenoliths, Shiva Publishing Ltd., Cheshire, 5-19.

[54] El-Akhal, H. (2004) Contribution to the Petrography, Geochemistry and Tectonic Setting of the Basalt Flows of the Umm-Qais Plateau, North Jordan. Geological Bulletin of Turkey, 47, 1-10.

[55] Thompson, R.N. (1987) Phase Equilibria Constraints on the Genesis and Magmatic Evolution of Oceanic Basalts. Earth Science Reviews, 24, 161-210. http://dx.doi.org/10.1016/0012-8252(87)90023-7 